\title{
NOX2 deficiency alters macrophage phenotype through an IL-10/STAT3 dependent mechanism: implications for traumatic brain injury
}

James P. Barrett ${ }^{1}$, Rebecca J. Henry ${ }^{1}$, Sonia Villapol ${ }^{2}$, Bogdan A. Stoica', Alok Kumar ${ }^{1}$, Mark P. Burns ${ }^{2}$, Alan I. Faden ${ }^{1}$ and David J. Loane ${ }^{1 *}$

\begin{abstract}
Background: NADPH oxidase (NOX2) is an enzyme system that generates reactive oxygen species (ROS) in microglia and macrophages. Excessive ROS production is linked with neuroinflammation and chronic neurodegeneration following traumatic brain injury (TBI). Redox signaling regulates macrophage/microglial phenotypic responses (pro-inflammatory versus anti-inflammatory), and NOX2 inhibition following moderate-tosevere TBI markedly reduces pro-inflammatory activation of macrophages/microglia resulting in concomitant increases in anti-inflammatory responses. Here, we report the signaling pathways that regulate NOX2-dependent macrophage/microglial phenotype switching in the TBI brain.

Methods: Bone marrow-derived macrophages (BMDMs) prepared from wildtype (C57BI/6) and NOX2 deficient $\left(\mathrm{NOX2}^{-/-}\right)$mice were treated with lipopolysaccharide (LPS; $\left.10 \mathrm{ng} / \mathrm{ml}\right)$, interleukin-4 (IL-4; $\left.10 \mathrm{ng} / \mathrm{ml}\right)$, or combined LPS/L-4 to investigate signal transduction pathways associated with macrophage activation using western immunoblotting and GPCR analyses. Signaling pathways and activation markers were evaluated in ipsilateral cortical tissue obtained from adult male wildtype and $\mathrm{NOX}^{-1-}$ mice that received moderate-level controlled cortical impact (CCI). A neutralizing anti-IL-10 approach was used to determine the effects of IL-10 on NOX2-dependent transitions from pro- to anti-inflammatory activation states.

Results: Using an LPS/IL-4-stimulated BMDM model that mimics the mixed pro- and anti-inflammatory responses observed in the injured cortex, we show that NOX2 $2^{-1-}$ significantly reduces STAT1 signaling and markers of proinflammatory activation. In addition, NOX2 ${ }^{-/-}$BMDMs significantly increase anti-inflammatory marker expression; IL-10-mediated STAT3 signaling, but not STAT6 signaling, appears to be critical in regulating this anti-inflammatory response. Following moderate-level CCI, IL-10 is significantly increased in microglia/macrophages in the injured cortex of NOX2 $2^{-/-}$mice. These changes are associated with increased STAT3 activation, but not STAT6 activation, and a robust anti-inflammatory response. Neutralization of IL-10 in NOX2 ${ }^{-1-}$ BMDMs or CCI mice blocks STAT3 activation and the anti-inflammatory response, thereby demonstrating a critical role for IL-10 in regulating NOX2-dependent transitions between pro- and anti-inflammatory activation states.

(Continued on next page)
\end{abstract}

\footnotetext{
* Correspondence: dloane@anes.umm.edu

${ }^{1}$ Department of Anesthesiology and Shock, Trauma and Anesthesiology

Research (STAR) Center, University of Maryland School of Medicine, 655 West

Baltimore Street, \#6-011, Baltimore, MD 21201, USA

Full list of author information is available at the end of the article
} 
(Continued from previous page)

Conclusions: These studies indicate that following TBI NOX2 inhibition promotes a robust anti-inflammatory response in macrophages/microglia that is mediated by the IL-10/STAT3 signaling pathway. Thus, therapeutic interventions that inhibit macrophage/microglial NOX2 activity may improve TBI outcomes by not only limiting pro-inflammatory neurotoxic responses, but also enhancing IL-10-mediated anti-inflammatory responses that are neuroprotective.

Keywords: NADPH oxidase, NOX2, Traumatic brain injury, Macrophage, Neuroinflammation, Interleukin-10,

\section{Background}

Macrophages play a critical role in regulating the innate immune response to acute and chronic inflammation, and they can change their activation state in response to growth factors and external cues [1]. In the central nervous system (CNS) following traumatic brain injury (TBI), resident microglia and infiltrating macrophages respond to the local injury; depending upon their activation state, they may contribute either to secondary injury or neurorestoration [2]. Macrophages exhibit a diverse array of activation phenotypes, each with different physiological and functional roles $[1,3,4]$. In vitro studies have demonstrated that pro-inflammatory (M1-like) activation is characterized by upregulation of inflammatory mediators such as nitric oxide synthase (NOS2) and pro-inflammatory cytokines (tumor necrosis factor $\alpha(\mathrm{TNF} \alpha)$, interleukin-1 $\beta$ (IL-1 $\beta))$ and is associated with production of reactive oxygen species (ROS) [1]. It can be induced by the pro-inflammatory cytokine interferon (IFN) $-\gamma$ and activation of toll-like receptors (TLR). IFN- $\gamma$ stimulation leads to phosphorylation of the transcription factor signal transducer activator of transcription (STAT)1, and effects of the TLR4 agonist, lipopolysaccharide (LPS), are mediated, in part, through STAT1 [5]. In contrast, anti-inflammatory (M2-like) macrophages are involved in both wound healing and resolution of inflammatory responses [4]. They are marked by expression of factors such as mannose receptor (CD206), arginase-1 (Arg1), and chitinase 3-like 3 (YM1). Stimulation of macrophages with interleukin-4 (IL-4) upregulates the expression of markers of the anti-inflammatory phenotype-effects mediated in part through activation of STAT6 [6]. Other inflammatory mediators such as interleukin-10 (IL-10) [7] and insulinstimulating growth factor-1 (IGF-1) [8] may also activate the anti-inflammatory phenotype.

Defining specific activation states in vivo is challenging due to the diversity of phenotypes, which are dependent on environmental and tissue-specific cues that allow microglia/macrophages to perform specific functions under different physiological and pathological conditions. The in vitro-defined phenotypic classification system (M1 versus M2 polarization) oversimplifies the activation spectrum that exists in humans and animal models [9] and does not describe the actual diversity of microglial/macrophage reactive states. This has led to serious questioning of the use of M1/M2 classification in microglia [10] and peripheral macrophages [11]. The actual mechanisms underlying phenotypic changes in microglia/macrophages are not fully elucidated, and an accepted nomenclature that describes such complex cellular responses is currently lacking. It has been demonstrated that the microglial/macrophage activation response to TBI is highly complex, with individual cells concurrently expressing both pro- and antiinflammatory phenotype markers in unique profiles that can be altered as a function of time, injury localization, and injury severity [12-14]. In the current study, we use general terms, pro-inflammatory, and anti-inflammatory, to describe microglial/macrophage phenotypic responses in the TBI brain.

NADPH oxidase (NOX2) is a multi-subunit enzyme complex responsible for the production of both intracellular and extracellular ROS by phagocytes, including microglia and macrophages [15]. NOX2 is the primary source of ROS generation in macrophages [16], and it participates in a wide range of cellular processes, including host defense, cell signaling, and cell differentiation. Excessive NOX2 activation is implicated in the progression of a number of neurodegenerative disorders [17-19]. Following a moderate-to-severe TBI NOX2 is chronically elevated in reactive macrophages/microglia surrounding the lesion for weeks to months postinjury $[20,21]$. NOX2 expression is also markedly increased in aged animals after TBI and is associated with increased pro-inflammatory activation and suppressed anti-inflammatory marker expression [22]. Notably, NOX2 inhibition reduces markers of pro-inflammatory activation, limits tissue loss and neurodegeneration, and improves neurological recovery in TBI mice [12, 23-26]. NOX2 inhibition also results in enhanced expression of antiinflammatory markers in macrophage/microglia [24, 27], and infiltrating macrophages are major contributors to the anti-inflammatory environment in the TBI brain in NOX2 knockout mice [24]. Based on accumulating pre-clinical evidence supporting NOX2-mediated neuroinflammation as an important therapeutic target for TBI, the goal of the current study was to elucidate the cell 
signaling mechanisms that regulate NOX2-dependent phenotypic changes in macrophages/microglia in the brain following TBI.

\section{Methods}

Animals

Studies were performed using either NOX2-deficient (NOX2 $^{-/-}$; B6.129S-Cybb ${ }^{\text {tm1Din } / J, ~ s t o c k ~ 002365 ; ~ J a c k s o n ~}$ Laboratories, Bar Harbor, ME,) adult male mice (1012 weeks old), or age-matched C57Bl/6 J (WT) male mice. Mice were housed in the Animal Care facility at the University of Maryland School of Medicine under a $12 \mathrm{~h}$ light-dark cycle, with ad libitum access to food and water. All surgical procedures were carried out in accordance with protocols approved by the Institutional Animal Care and Use Committee (IACUC) at the University of Maryland School of Medicine.

\section{Controlled cortical impact}

Our custom-designed controlled cortical impact (CCI) injury device consists of a microprocessor-controlled pneumatic impactor with a $3.5-\mathrm{mm}$ diameter tip as previously described [28]. Briefly, mice were anesthetized with isoflurane evaporated in a gas mixture containing $70 \% \mathrm{~N}_{2} \mathrm{O}$ and $30 \% \mathrm{O}_{2}$ and administered through a nose mask. Mice were placed on a heated pad, and core body temperature was maintained at $37{ }^{\circ} \mathrm{C}$. The head was mounted in a stereotaxic frame, a $10-\mathrm{mm}$ midline incision was made over the skull, and the skin and fascia were reflected. A 5-mm craniotomy was made on the central aspect of the left parietal bone. The impounder tip of the injury device was then extended to its full stroke distance $(44 \mathrm{~mm})$, positioned to the surface of the exposed dura, and reset to impact the cortical surface. Moderate-level CCI was induced using an impactor velocity of $6 \mathrm{~m} / \mathrm{s}$ and deformation depth of $2 \mathrm{~mm}$. After injury, the incision was closed with interrupted $6-0$ silk sutures, anesthesia was terminated, and the animal was placed into a heated cage to maintain normal core temperature for $45 \mathrm{~min}$ post-injury. Sham animals underwent the same procedure as CCI mice except for the impact.

Intracerebroventricular (i.c.v.) guide cannula implantation and osmotic pump infusion

Prior to CCI, the right lateral ventricle of the mouse was stereotaxically perforated with a brain infusion kit 3 (ALZET, DURET Corporation, Cupertino, CA, USA; coordinates: $0.7 \mathrm{~mm}$ posterior to the bregma, $1.5 \mathrm{~mm}$ lateral to the bregma, $2 \mathrm{~mm}$ deep). Immediately following $\mathrm{CCI}$ on the left parietal cortex the infusion cannula was connected to an osmotic minipump (ALZET; pump model: 1007D) that was implanted subcutaneously (sc) in the animal's back, just behind scapula. Osmotic pumps were primed for approximately $8 \mathrm{~h}$ prior to implantation and were either filled with $1 \mathrm{mg} / \mathrm{ml}$ antiIL-10 neutralizing antibody ( $\alpha$ IL-10; clone JES5-16E3; eBioscience, San Diego, CA) or equal concentration isotype control rat $\alpha \operatorname{IgG} 2 \mathrm{~b} \mathrm{~K}$ (clone Eb149/10h5 eBioscience). Once implanted, the pumps continually infused $\alpha \mathrm{IL}-10$ or control $\alpha \operatorname{IgG} 2 \mathrm{~b} \mathrm{~K}$ into the lateral ventricle for 3 days at a rate of $0.5 \mu \mathrm{L} / \mathrm{h}$.

\section{Study 1}

Sham $(n=5)$ and CCI $(n=5)$ of WT and NOX2 ${ }^{-1-}$ mice were anesthetized $(100 \mathrm{mg} / \mathrm{kg}$ sodium pentobarbital, I.P.) at 1,3 , and 7 days post-injury and transcardially perfused with ice-cold $0.9 \%$ saline $(100 \mathrm{ml})$. Ipsilateral cortical tissue was rapidly dissected and was snap-frozen on liquid nitrogen for RNA extraction.

\section{Study 2}

Sham $(n=5)$ and CCI $(n=5)$ of WT and NOX2 $2^{-/-}$mice were anesthetized $(100 \mathrm{mg} / \mathrm{kg}$ sodium pentobarbital, I.P.) at 7 days post-injury and transcardially perfused with ice-cold $0.9 \%$ saline $(100 \mathrm{ml})$, followed by $300 \mathrm{ml}$ of $4 \%$ paraformaldehyde. Brains were removed and postfixed in $4 \%$ paraformaldehyde overnight and were cryoprotected in 30\% sucrose for histological analysis.

\section{Study 3}

$\alpha \mathrm{IL}-10(1 \mathrm{mg} / \mathrm{ml})$ or isotype control $\alpha \operatorname{IgG} 2 \mathrm{~b} \mathrm{~K}$ was delivered i.c.v. via osmotic pump infusion to WT and $\mathrm{NOX}^{-1-}$ CCI mice ( $n=6 /$ group). The dose of $\alpha \mathrm{IL}-10$ was chosen based on prior studies demonstrating neutralization of IL-10 in a mouse model of spinal cord injury (SCI) [29]. Sham WT mice $(n=6)$ were used as control for baseline mRNA expression levels. Animals were anesthetized $(100 \mathrm{mg} / \mathrm{kg}$ sodium pentobarbital, I.P.) at 3 days post-injury and transcardially perfused with ice-cold $0.9 \%$ saline $(100 \mathrm{ml})$. Ipsilateral cortical tissue was rapidly dissected and was snap-frozen on liquid nitrogen for RNA extraction.

\section{Preparation and culture of bone marrow-derived macro- phages (BMDMs)}

BMDMs were isolated from the marrow of the femurs and tibias of uninjured adult WT and $\mathrm{NOX}^{-1-}$ male mice as previously described [8]. Briefly, following terminal anesthesia $(100 \mathrm{mg} / \mathrm{kg}$ sodium pentobarbital, I.P.), the marrow was flushed out into a sterile falcon tube in Dulbecco's modified Eagle's medium (DMEM; Gibco Invitrogen, Carlsbad, CA) supplemented with heatinactivated fetal bovine serum (FBS; 10\%; Atlanta Biologicals, Flowery Branch, GA) and penicillin/streptomycin (1\%; Gibco Invitrogen) to obtain a single cell suspension. The suspension was centrifuged $(400 \times g, 5 \mathrm{~min})$, the supernatant was discarded, and the resulting pellet was 
resuspended in $20 \mathrm{ml}$ of DMEM supplemented with Ladmac-conditioned media (20\%). Cells were seeded in sterile cell culture flasks (T75 $\mathrm{cm}^{2}$ flasks) and were maintained in culture for a further 6 days, with media being replaced on day 4 . On day 6 , cells were transferred to 24-well plates $\left(0.4 \times 10^{6}\right.$ cells per well) or 96-well plates $\left(0.5 \times 10^{5}\right.$ cells per well) and remained in culture for a further 2 days.

\section{Treatment of BMDMs}

Cells were incubated in the presence of both LPS (10 ng/ml; Sigma-Alrich, St. Louis, MO) and IL-4 (10 ng/ml; R\&D Systems, Minneapolis, MN), supernatants were collected for analysis of cytokines by ELISA, and cells were harvested for analysis of markers of macrophage activation by real-time PCR and polyacrylamide gel electrophoresis followed by western immunoblotting. To investigate IL-10-mediated effects in antiinflammatory activation, cells were treated with LPS/IL4 in the presence or absence of a neutralizing antibody to $\alpha \mathrm{IL}-10(10 \mu \mathrm{g} / \mathrm{ml}$; eBioscience) or the appropriate isotype control (rat $\alpha \operatorname{IgG} 2 \mathrm{~b} \mathrm{~K}$; eBioscience).

\section{Real-time PCR}

Total RNA was extracted from BMDMs and snap-frozen sham and TBI cortical tissue of WT and $\mathrm{NOX}^{-/-}$mice using an RNeasy isolation kit (Qiagen, Valencia, CA) with on-column DNase treatment (Qiagen). cDNA synthesis was performed using a Verso cDNA RT kit (Thermo Scientific, Pittsburg, PA); the protocols used were according to the manufacturer's instructions. Realtime PCR was performed using TaqMan gene expression assays (Ym1, Mm00657889_m1; Arg1, Mm00475988_m1; SOCS3, Mm00545913_s1; IL-4R $\alpha, \quad M m 00446186 \_m 1 ;$ TGF- $\beta$, Mm00441724_m1; SHIP1, Mm00494987_m1; and GAPDH Mm99999915_g1; Applied Biosystems, Carlsbad, CA) on an ABI 7900 HT FAST Real-Time PCR machine (Applied Biosystems). Samples were assayed in duplicate in one run (40 cycles), which was composed of 3 stages, $50{ }^{\circ} \mathrm{C}$ for $2 \mathrm{~min}, 95{ }^{\circ} \mathrm{C}$ for $10 \mathrm{~s}$ for each cycle (denaturation) and finally the transcription step at $60^{\circ} \mathrm{C}$ for $1 \mathrm{~min}$. Gene expression was calculated relative to the endogenous control sample (GAPDH) to determine relative expression values, using the $2-\Delta \Delta \mathrm{Ct}$ method (where $\mathrm{Ct}$ is the threshold cycle).

\section{Western blotting}

Proteins from BMDMs and ipsilateral cortical tissue were extracted using RIPA buffer, equalized, and loaded onto 5-20\% gradient gels for SDS PAGE (Bio-Rad; Hercules, CA). Proteins were transferred onto nitrocellulose membranes and then blocked for $1 \mathrm{~h}$ in $5 \%$ milk in $1 \times$ TBS containing $0.05 \%$ Tween-20 (TBS-T) at room temperature. The membrane was incubated in mouse anti-arginase $1(\mathrm{~N}-20)$ (1:1000; BD Transduction Laboratories, San Jose, CA), rabbit anti-STAT6 (1:1000; Cell signaling, Danvers, MA), rabbit anti-pSTAT6 (1:1000; Cell signaling), mouse anti-STAT3 (1:1000; Cell signaling), rabbit anti-pSTAT3 (1:1000; Cell signaling), or mouse anti- $\beta$-Actin (1:5000; Sigma-Aldrich) overnight at $4{ }^{\circ} \mathrm{C}$, then washed three times in TBS-T, and incubated in appropriate HRP-conjugated secondary antibodies (Jackson ImmunoResearch Laboratories, West Grove, PA) for $2 \mathrm{~h}$ at room temperature. Membranes were washed three times in TBS-T, and proteins were visualized using SuperSignal West Dura Extended Duration Substrate (Thermo Scientific, Rockford, IL). Chemiluminescence was captured ChemiDoc ${ }^{\mathrm{Tn}}$ XRS+ System (Bio-Rad), and protein bands were quantified by densitometric analysis using BioRad Molecular Imaging Software. The data presented reflects the intensity of target protein band normalized based on the intensity of the endogenous control for each sample (expressed in arbitrary units).

\section{Cytokine and nitric oxide analysis}

Concentrations of TNF $\alpha$ (R\&D Systems; DY410), IL-6 (R\&D Systems; DY406), and IL-10 (R\&D Systems; DY41705) were measured in supernatant samples obtained from BMDMs by ELISA. Briefly, standards or samples $(100 \mu \mathrm{l})$ were added to antibody-coated 96well plates and incubated for $2 \mathrm{~h}$ at room temperature, plates were washed and samples were incubated in detection antibody for $2 \mathrm{~h}$. Plates were washed and incubated in horseradish peroxidaseconjugated streptavidin for $20 \mathrm{~min}$ at room temperature. Substrate solution (tetramethylbenzidine; Sigma-Alrich) was added, incubation continued at room temperature in the dark for $30 \mathrm{~min}$, and the reaction was stopped using $\mathrm{H}_{2} \mathrm{SO}_{4}(1 \mathrm{M})$. Absorbance measurements were read at $450 \mathrm{~nm}$ using a Synergy HT Multi-Mode Microplate Reader (Biotek, Winooski, VT). Cytokine concentrations were calculated relative to the appropriate standard curve and expressed as pg cytokine/ $\mu \mathrm{g}$ of protein. Nitric oxide (NO) release was assayed using a Greiss reagent assay (Invitrogen; G7921), per the manufacturer's instructions. NO concentrations were calculated using standard curves generated from a nitrite stock, and results were expressed in micromoles.

\section{IL-10 fluorescent in situ hybridization (FISH),} immunohistochemistry, and quantification of IL-10 co-localization

$20 \mu \mathrm{m}$ coronal brain sections were cut on a cryostat, mounted on gelatin-coated glass slides (Superfrost Plus, Fisher Scientific, Tusin, CA), and stored at $-80{ }^{\circ} \mathrm{C}$ until use. Fluorescent in situ hybridization (FISH) was 
performed as per the manufacturer's instructions using RNAscope ${ }^{\circ}$ Technology 2.0 Red Fluorescent kit for Fresh Frozen Tissue (Advanced Cell Diagnostics, Inc., Hayward, CA). Tissue sections were dehydrated by 50,70 , and $100 \%$ ethanol gradually for $5 \mathrm{~min}$, and then boiled for 3-5 min with pretreatment 2 solution, incubated with pretreatment 3 solution, and incubated with target IL-10 probe (Mus musculus interleukin 10 (IL-10) mRNA; accession number NM_010548.2, target region 2-1095) at $40{ }^{\circ} \mathrm{C}$ for $2 \mathrm{~h}$ in the HybEZ humidified incubator. A dapB probe targeting a bacterial gene was used as a negative control and Ppib (Mus musculus peptidylprolyl isomerase B mRNA; accession number NM_011149.2, target region 98-856) was used as a positive control. After FISH, slides were washed three times with PBS and blocking with PBS containing 0.5\% Triton $\mathrm{X}-100$ and $2 \%$ normal goat serum for $1 \mathrm{~h}$. Immunohistochemistry was performed using polyclonal anti-rabbit Iba-1 (1:500, Wako Chemicals, Richmond, VA) incubated overnight at $4{ }^{\circ} \mathrm{C}$. Alexa Fluor 488-conjugated goat anti-rabbit IgG (1:1000, Invitrogen, Carlsbad, CA) was applied for $1 \mathrm{~h}$ at room temperature. Sections were rinsed with PBS three times and incubated in PBS with DAPI solution (1:50.000) for counterstained nuclei. The sections were washed three times in distilled water and coverslipped with Fluoro-Gel with Tris Buffer mounting medium (Electron Microscopy Sciences, Hatfield, PA). Experiments were repeated three times.

All analyses were performed on digital images captured using a $\times 20$ objective in a sampled region within the ipsilateral primary somatosensory cortex with a field of $151.894 \mathrm{~mm}^{2}$ around the impact site. Two to three fields were randomly selected per brain section (see Fig 6 B1) between -1.70 and $-2.30 \mathrm{~mm}$ from the bregma, and a total of 2 to 4 brain sections per brain. Images were taken in the cortical layers III-IV for sham mice. IL-10 positive cells (red) that colocalized with DAPI nuclei (blue) were manually counted. The negative control probe did not contain any stained cells. Stained cells were examined under a florescent microscope (Axioplan 2 Zeiss) and were analyzed using Image J software (http://rsbweb.nih.gov/ij/). RNA positive cells were quantitated in each cortical sampling zone, each field of view analyzed was the sum of three images of sequential optical Z-stack images combined into a single overlay image, and IL-10 positive cells were counted using the overlay. The result from all the fields of view in a given animal was averaged to obtain the value for that individual ( $n=5$ /group). Double positive cells were identified using the IL-10 probe and colocalization with Iba-1 (green) were based on analysis of microscopic fields of view as previously described [30], keeping all acquisition parameters constant. The percentage of mRNA IL-10 positive cells that colocalized with Iba- 1 immunoreactive cells relative to the total number of IL-10 positive cells was assessed. A confocal microscope (Leica SP8) was used to take images from FISH-ISH brain sections using $\times 20, \times 40$, and $\times 63$ objectives.

\section{Statistical analysis}

Randomization protocols were employed in all experiments, and individuals performing analysis were blinded to treatment and genotype groups. Data are reported as the mean \pm SEM, and the number of experiments is indicated in each case. Statistical analysis was carried out using a two- or three-way analysis of variance (ANOVA) with post hoc Bonferroni tests or one-way ANOVA followed by post hoc Newman-Keuls analysis to identify specific differences between groups. When comparisons were being made between two conditions, an unpaired Student's $t$ test was performed. Significance level was set as $P<0.05$.

\section{Results \\ LPS/L-4 induces a mixed phenotype in BMDMs}

It is well documented that macrophages/microglia adopt distinct phenotypes in response to inflammatory stimuli such as LPS (pro-inflammatory) or IL-4 (anti-inflammatory) [31, 32], however, recently it has been observed that after TBI, macrophages/microglia express markers associated with both pro- and antiinflammatory phenotypes [12-14]. To mimic this activation spectrum in vitro, BMDMs were incubated in the presence of both LPS $(10 \mathrm{ng} / \mathrm{ml})$ and IL-4 $(10 \mathrm{ng} / \mathrm{ml})$, and this combined treatment significantly upregulated both pro- and anti-inflammatory activation markers. Specifically, LPS/IL-4 stimulation led to a significant increase in the supernatant concentration of TNF $\alpha(P<0.001$; Student's $t$ test; Fig. 1a) and IL-6 $(P<0.001$; Student's $t$ test; Fig. $1 \mathrm{~b})$, both of which are cytokines associated with a pro-inflammatory phenotype. We also observed elevated levels of nitrite $(P<$ 0.001; Student's $t$ test; Fig. 1c), an indication of increased NO production. The changes in proinflammatory markers were also accompanied by an increase in Arg1 protein $(P<0.001$; Student's $t$ test; Fig. 1d), and an upregulation in YM1 mRNA expression $(P<0.001$; Student's $t$ test; Fig. 1f), both markers of anti-inflammatory macrophages. Of note, when we stimulated BMDMs with only LPS or IL-4 alone, there was increased expression of markers of proand anti-inflammatory phenotypes, respectively, but mixed pro- and anti-inflammatory phenotype markers were not detected (data not shown). These results demonstrate that LPS/IL-4 stimulation of BMDMs produces a mixed pro- and anti-inflammatory activation state, similar to the activation profile observed in the TBI brain. 

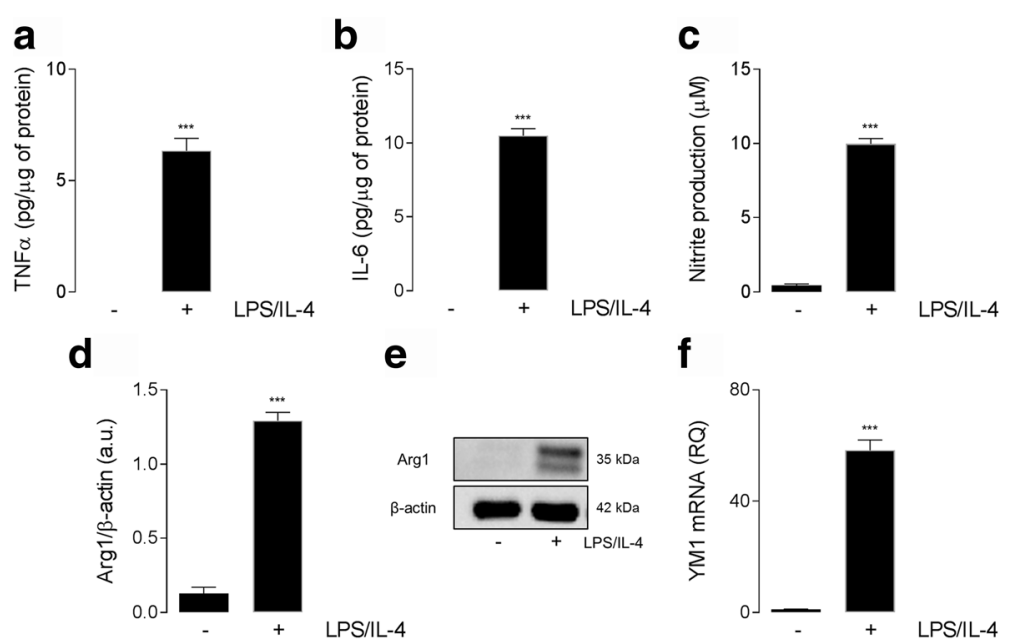

Fig. 1 LPS/L-4 induces a mixed phenotype in BMDMs. (a-f) LPS/L-4 (both $10 \mathrm{ng} / \mathrm{ml} ; 24 \mathrm{~h}$ ) stimulation significantly increased the production of TNFa (a), IL-6 (b), and Nitrite (b) in BMDMs from WT mice ${ }^{* * *} P<0.001$; Student's $t$ test). LPS/L-4 increased Arg1 (d) protein expression (representative western immunoblot shown (e)) and YM1 (f) mRNA expression in BMDMs ( ${ }^{* * *} P<0.001$; Student's $t$ test). All data are expressed as means $( \pm$ SEM; $n=3)$

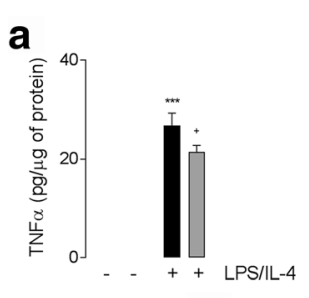

d
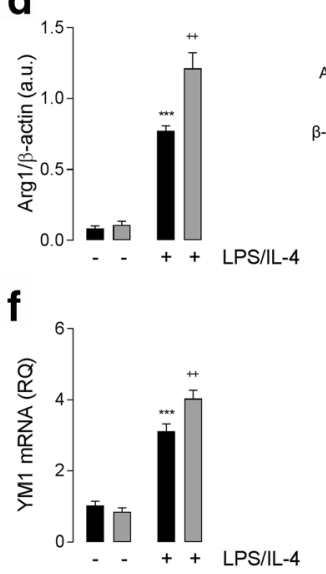

\section{b}

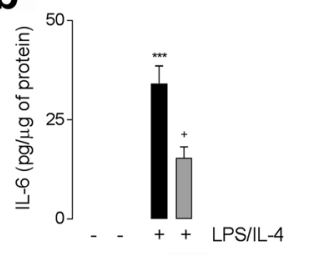

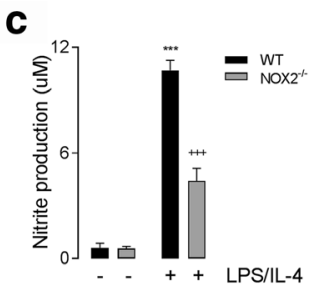

e
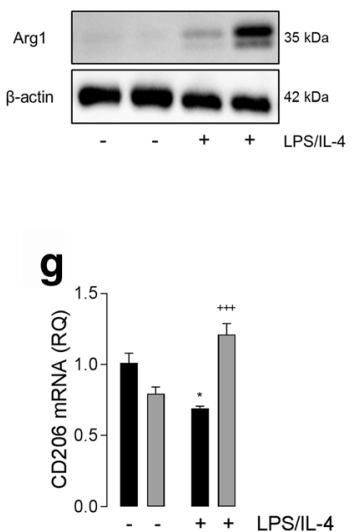

Fig. 2 NOX2 deficiency alters macrophage response to LPS/LL-4. LPS/LL-4 (both $10 \mathrm{ng} / \mathrm{ml} ; 24 \mathrm{~h}$ ) stimulation significantly increased the release of TNFa (a), IL-6 (b), and Nitrite (c) in WT and NOX2 ${ }^{-/-}$BMDMs ( ${ }^{* * *} P<0.001$; ANOVA). LPS/L-4-induced increase in pro-inflammatory markers was significantly reduced in and NOX2 ${ }^{-/-}$BMDMs compared with WT BMDMs $\left({ }^{++} P<0.01,{ }^{+++} P<0.001\right.$, WT LPS/IL-4 Vs. NOX2 ${ }^{-/-}$LPS/IL-4; ANOVA). LPS/IL-4

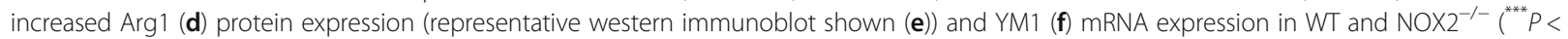
0.001; ANOVA). Expression of anti-inflammatory markers was significantly increased in NOX2 ${ }^{-/-}$BMDMs compared with WT BMDMs $\left({ }^{++} P<0.01\right.$, ${ }^{+++} P<0.001$, WT LPS/LL-4 vs. NOX2 $2^{-/}$LPS/LL-4; ANOVA). LPS/IL-4 significantly decreased CD206 mRNA expression in WT BMDMs ( ${ }^{*} P<0.05 ;$ ANOVA; g), while CD206 mRNA expression was significantly increased in NOX2 ${ }^{-/-}$BMDMs following LPS/IL-4 stimulation $\left(^{+++} P<0.001\right.$, NOX2 $2^{-/-}$con vs. NOX2 - LPS/IL-4; ANOVA). All data are expressed as means ( \pm SEM; $n=6)$ 
NOX2 deficiency alters macrophage response to LPS/LL-4 Given that $\mathrm{NOX}^{-/-}$TBI mice exhibit enhanced antiinflammatory activation that is associated with improved outcomes following TBI [24], we set out to investigate mechanisms of NOX2-dependent macrophage phenotype switching using the LPS/IL-4 stimulation model. WT and NOX2 ${ }^{-/-}$BMDMs were treated with LPS/IL-4 for $24 \mathrm{~h}$, and pro and anti-inflammatory activation markers were assessed. LPS/IL-4 significantly increased supernatant concentration of TNF $\alpha(P<0.001$; ANOVA; Fig. 2a), IL-6 $(P<0.001$; ANOVA; Fig. $2 b)$, and nitrite $(P$ $<0.001$; ANOVA; Fig. 2c) in WT BMDMs. In contrast, levels of each pro-inflammatory marker were significantly reduced in LPS/IL-4 stimulated $\mathrm{NOX}^{-/-}$ BMDMs $\quad(P<0.05 \quad$ [TNF $\alpha, \quad$ IL-6]; $P<0.001$ [nitrite]; ANOVA; Fig. 2a-c). In addition, LPS/IL-4 significantly increased Arg1 protein $(P<0.001$; ANOVA; Fig. $2 \mathrm{~d})$ and YM1 mRNA $(P<0.001$; ANOVA; Fig. $2 \mathrm{f})$ expression in WT BMDMs; expression of both anti-inflammatory markers were significantly increased beyond WT levels in NOX2 ${ }^{-/-}$cells $(P<0.01$; ANOVA; Fig. $2 d, f)$. CD206 is another anti-inflammatory activation marker, and LPS/IL-4 stimulation led to a significant decrease in CD206 mRNA expression in WT BMDMs $(P<0.05$; ANOVA; Fig. 2g). In contrast, LPS/IL-4 significantly increased CD206 mRNA expression in $\mathrm{NOX}^{-/-}$ BMDMs $(P<0.001$; ANOVA; Fig. $2 \mathrm{~g})$.

\section{STAT1 activation is attenuated in NOX2 $2^{-/-}$BMDMs, whereas STAT6 activation is unchanged}

Having observed genotype-related changes in the expression of markers associated with the pro- and anti- inflammatory phenotypes, we set out to investigate changes in signaling pathways involved in the induction of these activation states. STAT1 is elevated following stimulation with pro-inflammatory cytokines and/or TLR agonists [5, 8]. Here, we demonstrate that STAT1 phosphorylation is significantly elevated in WT and $\mathrm{NOX}^{-/-}$BMDMs in response to LPS/IL-4 stimulation $(P<0.001$; ANOVA; Fig. 3a, c). However, STAT1 activation was significantly reduced in LPS/IL-4 treated NOX2 -I- cells when compared to WT cells $(P<0.05$; ANOVA; Fig. 3c). STAT6 is a part of the IL-4 signaling cascade $[6,33]$ and having observed enhanced expression of anti-inflammatory markers in $\mathrm{NOX}^{-/-}$cells we anticipated that these changes were mediated by STAT6 activation. LPS/IL-4 stimulation significantly increased pSTAT6 expression in WT and $\mathrm{NOX}^{-/-}$BMDMs $(P<0.001$; ANOVA; Fig. 3b, d), however, there was no genotyperelated difference in STAT6 activation.

\section{IL-10 and STAT3 signaling are increased in LPS/IL-4 stimulated $\mathrm{NOX2}^{-/-}$BMDMs}

We demonstrated that the LPS/IL-4-mediated increase in anti-inflammatory markers in $\mathrm{NOX}^{-/-}$BMDMs was independent of STAT6 activation, therefore, we set out to determine what signaling pathways were involved in the NOX2-dependent shift towards an anti-inflammatory phenotype. We next measured supernatant concentration of IL-10 in LPS/IL-4-treated cells; there was a significant increase in IL-10 production in WT and $\mathrm{NOX}_{2}{ }^{-/}$BMDMs $(P<0.001$; ANOVA; Fig. 4a), and this effect was greater in $\mathrm{NOX}^{-1-}$ cells when compared to WT cells $(P<0.001$; ANOVA; Fig. 4a). We also observed an increase in
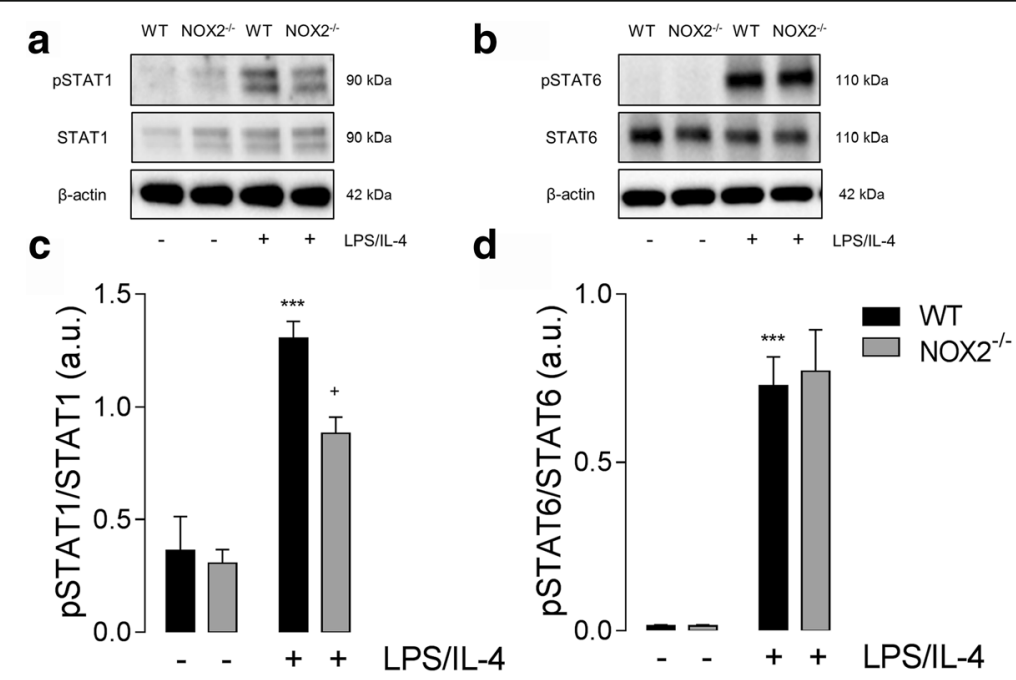

Fig. 3 STAT1 activation is attenuated in NOX2 ${ }^{-1-}$ BMDMs, whereas STAT6 activation is unchanged. (a, b) Protein expression of phosphorylated STAT1 and phosphorylated STAT6 in response to LPS/L-4 stimulation was assessed by western immunoblotting. (c) LPS/LL-4 (both $10 \mathrm{ng} / \mathrm{ml}$; $24 \mathrm{~h}$ ) increased pSTAT1 expression ( ${ }^{* *} P^{*}<0.001$, ANOVA) in WT BMDMs. In contrast, LPS/L-4-induced increase in pSTAT1 was significantly reduced in NOX2 $2^{-1-}$ BMDMs $\left({ }^{+} P<0.05\right.$, WT LPS/LL-4 Vs. NOX2 $2^{-1-}$ LPS/IL-4; ANOVA). (d) LPS/IL-4 significantly increased pSTAT6 $\left(^{* * *} P<0.001 ;\right.$ ANOVA) in WT and $\mathrm{NOX}^{-/-}$BMDMs, but no genotype related differences were observed. All data are expressed as means $( \pm \operatorname{SEM} n=5)$ 


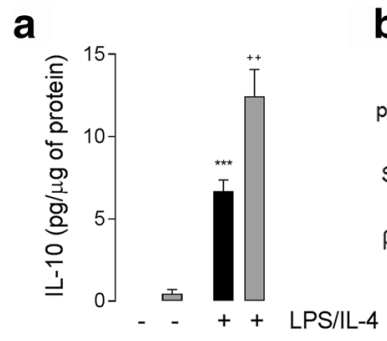

b

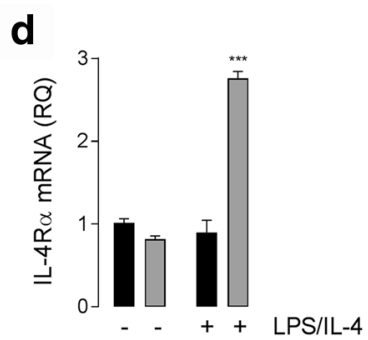

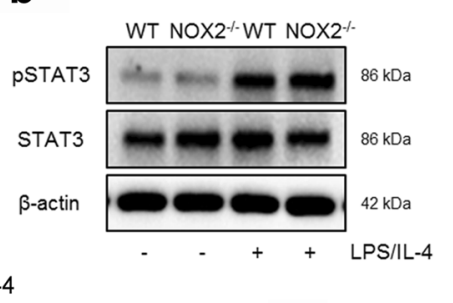

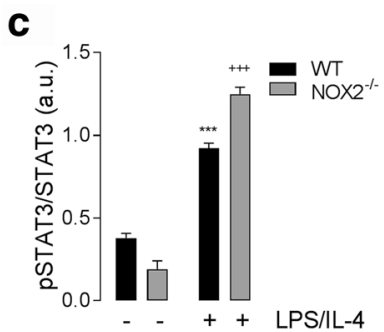

e

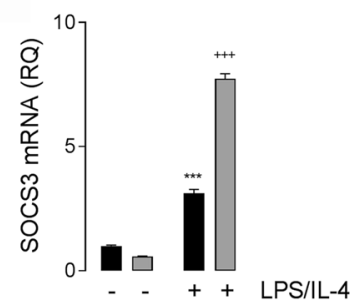

Fig. 4 IL-10 production and downstream effectors are elevated in NOX2 ${ }^{-/-}$BMDMs in response to LPS/L-4. (a) LPS/L-4 (both $10 \mathrm{ng} / \mathrm{ml} ; 24 \mathrm{~h}$ ) stimulation

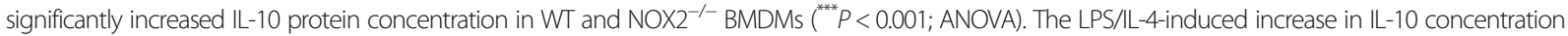

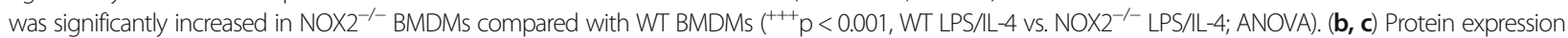
of phosphorylated STAT3 was significantly increased in WT and NOX2 ${ }^{-/-}$BMDMs following LPS/L-4 stimulation ( ${ }^{* * *} P<0.001$; ANOVA; representative western immunoblot shown in b). LPS/L-4-induced pSTAT3 expression was significantly increased in NOX2 ${ }^{-1-}$ BMDMs compared with WT BMDMs ${ }^{+++} P<0.001$, WT LPS/LL-4 vs. NOX2 $2^{-1-}$ LPS/L-4; ANOVA). (d) LPS/IL-4 significantly increased IL-4Ra mRNA expression NOX2 ${ }^{-/-}$BMDMs ( ${ }^{* * *} P<0.001$; ANOVA). (e) SOCS3 mRNA expression was significantly increased in WT and NOX2 ${ }^{-/-}$BMDMs following LPS/L-4 stimulation ( ${ }^{* * *} P<0.001 ;$ ANOVA). LPS/L-4-induced SOCS3 expression

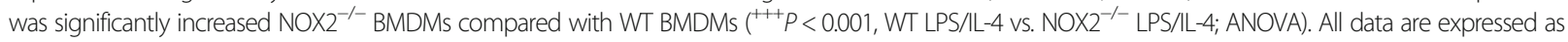
means $( \pm \mathrm{SEM}, n=6)$

STAT3 phosphorylation in WT and $\mathrm{NOX}^{-/-}$cells $(P<0.001$; ANOVA; Fig. 4b, c). Notably, STAT3 activation was significantly increased in LPS/IL-4 treated $\mathrm{NOX} 2^{-/-}$ BMDMs when compared to WT BMDMs $(P<0.001$; ANOVA; Fig. 4c). We then analyzed the expression of inflammatory markers downstream of IL-10 such as IL-4R $\alpha$ and SOCS3 [34]. Consistent with the activation of IL-10 signaling there was a significant increase in IL-4R $\alpha$ $(P<0.001 ;$ ANOVA; Fig. $4 \mathrm{~d})$ and SOCS3 $(P<0.001$; ANOVA; Fig. 4e) mRNA expression in NOX2 $2^{-/-}$BMDMs when compared to levels in WT BMDMs.

\section{Increased anti-inflammatory activation in NOX2 $2^{-/-}$ \\ BMDMs is mediated through IL-10}

In order to test whether IL-10 mediates the antiinflammatory shift in NOX2 $2^{-/-}$macrophages, WT and $\mathrm{NOX}^{-/-}$BMDMs were stimulated with LPS/IL-4 in the presence or absence of neutralizing $\alpha \mathrm{IL}-10$. As previously observed, LPS/IL-4 treatment significantly increased STAT3 activation in WT BMDMs $(P<0.01$; ANOVA; Fig. 5a, b); an effect that was significantly increased in NOX2 $2^{-1-}$ BMDMs $(P<0.05$; ANOVA; Fig. 5b). In the presence of $\alpha$ IL-10, pSTAT3 expression was significantly reduced in $\mathrm{NOX}^{-/-}$BMDMs $(P<0.001$; ANOVA; Fig. 5b), and the genotype effect was lost. Analysis of STAT6 activation confirmed equal pSTAT6 expression in WT and NOX2 $2^{-/-}$BMDMs following LPS/ IL-4 stimulation $(P<0.001$; ANOVA; Fig. 5a, c). IL-10 neutralization significantly decreased STAT6 activation in both genotypes $\left(P<0.01[\mathrm{WT}] ; P<0.001\left[\mathrm{NOX}^{-/-}\right.\right.$]; ANOVA; Fig. 5c), but STAT6 activation remained significantly elevated when compared to control unstimulated cells. We next assessed Arg1 (Fig. 5a, d), IL-4R $\alpha$ (Fig. 5e), SOCS3 (Fig. 5f), and IL-10 (Fig. 5g) expression in this experimental paradigm, and as predicted, there was a significant increase in each marker in $\mathrm{NOX}^{-/-}$ BMDMs when compared to WT BMDMs $(P<0.05$ [Arg1; SOCS3; IL-10]; $P<0.001$ [IL-4R $\alpha$ ]; ANOVA; Fig. $5 d-g)$. In the presence of $\alpha I L-10$, the genotype effects on Arg1 and IL-4R $\alpha$ expression were lost, and levels were returned to that of unstimulated cells $(P<$ 0.001; ANOVA; Fig. 5d, e). LPS/IL-4-induced SOCS3 and IL-10 mRNA expression was increased in the presence of $\alpha \mathrm{IL}-10(P<0.001, P<0.01$; ANOVA; Fig. 5f, g); however, the genotype-related effect on SOCS3 was lost when IL-10 was neutralized by $\alpha \mathrm{IL}-10$ (Fig. 5f).

NOX2 deficiency results in increased cortical IL-10 expression following controlled cortical impact

Having demonstrated that IL-10/STAT3 signaling mediates the genotype-related changes in anti-inflammatory activation in $\mathrm{NOX}^{-/-} \mathrm{BMDMs}$, we then set out to evaluate genotype related changes in IL-10 expression in the TBI brain using a moderate-level CCI model. IL-10 mRNA expression was measured in ipsilateral cortex of sham and TBI WT and NOX2 $2^{-1-}$ mice at 24 and $72 \mathrm{~h}$ 

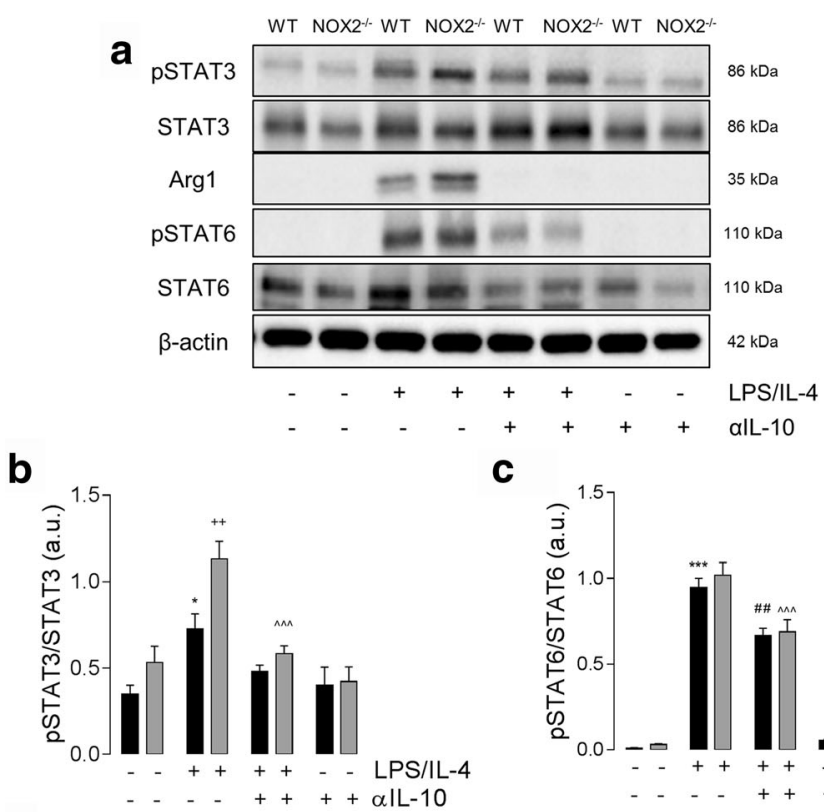

C
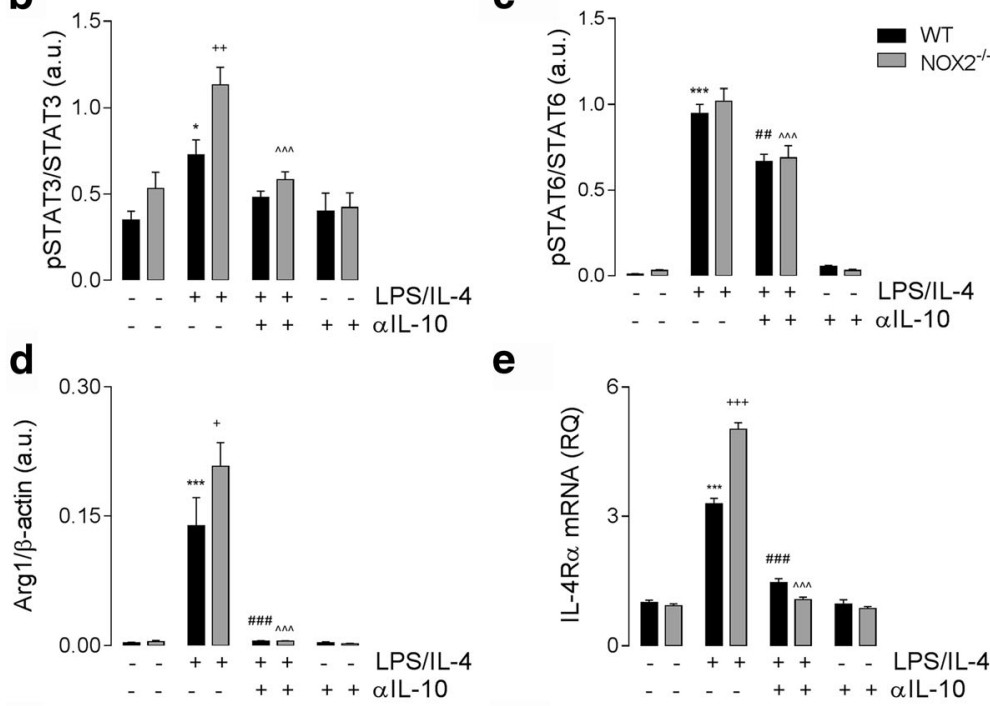

e
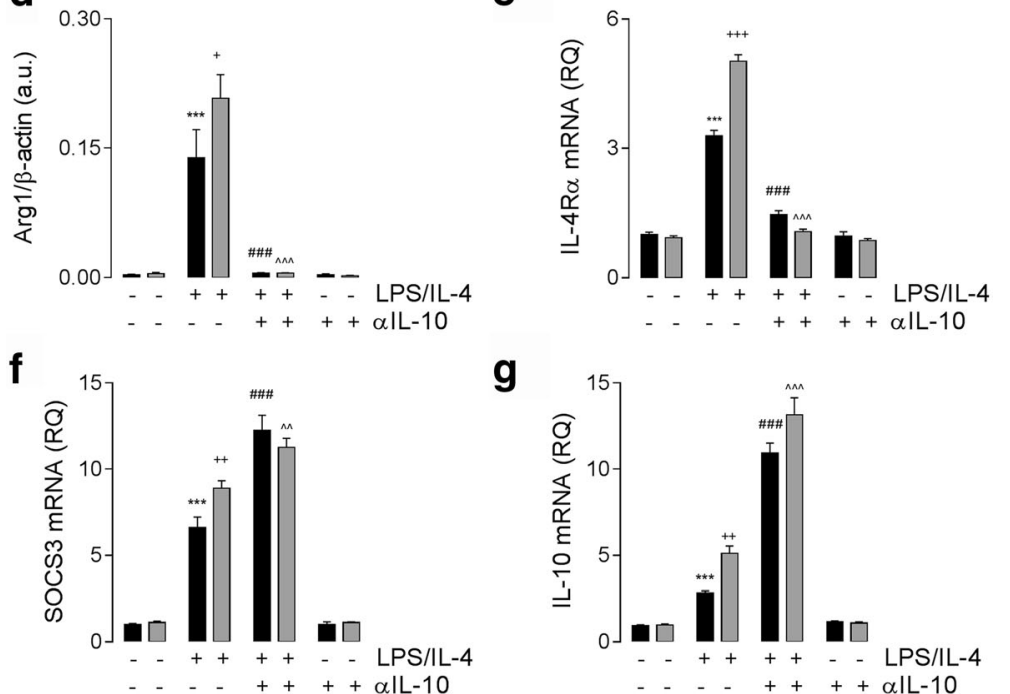

Fig. 5 Increased anti-inflammatory activation in NOX2 ${ }^{-1-}$ BMDMs is mediated through IL-10. (a) Representative immunoblots for pSTAT3, STAT3, Arg1, pSTAT6, STAT6, and $\beta$-actin. (b) LPS/L-4 (both $10 \mathrm{ng} / \mathrm{ml} ; 24 \mathrm{~h}$ ) increased the expression of pSTAT3 in WT BMDMs ( ${ }^{*} P<0.05$, vs. control; ANOVA), and LPS/L-4-induced pSTAT3 expression was significantly increased in NOX2 ${ }^{-/-}$BMDMs (+ ${ }^{+} P<0.01$, WT LPS/L-4 vs. NOX2 ${ }^{-/-}$LPS/L-4; ANOVA). Co-incubation of BMDMs with neutral-

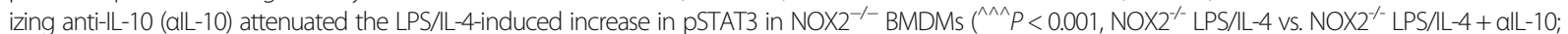
ANOVA). (c) all-10 attenuated the LPS/L-4-induced increase pSTAT6 expression ( $P<0.001$, control vs. LPS/L-4; ${ }^{* \#} P<0.01$, WT LPS/IL-4 vs. WT LPS/L-4 + alL-10; ${ }^{\wedge \wedge \wedge} P<0.001$, NOX2 ${ }^{--}$LPS/L-4 vs. NOX2 ${ }^{-/-}$LPS/LL-4 + alL-10; ANOVA), and no genotype-related changes were observed. (d, e) LPS/L-4 induced an increase in Arg1 (d) protein expression, and IL-4Ra (e) mRNA expression in WT and NOX2 $2^{-1-}$ BMDMs $\left({ }^{* * *} P<0.001\right.$, vs. control; ANOVA); these effects were significantly increased in NOX2 ${ }^{-1-}$ BMDMs $\left({ }^{+} P<0.05,{ }^{+++} P<0.001\right.$, WT LPS/LL-4 Vs. NOX2 ${ }^{-1-}$ LPS/LL-4; ANOVA). The LPS/LL-4 mediated effects on Arg1 and IL-4Ra expression

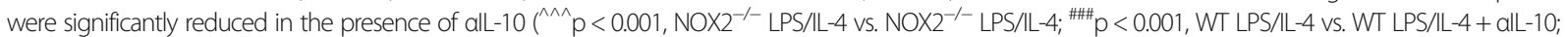
ANOVA). (f, g) SOCS3 and IL-10 mRNA expression was significantly increased in WT and NOX2 ${ }^{-1-}$ BMDMs following LPS/L-4 stimulation $\left({ }^{*} P<0.05,{ }^{* * *} P<0.001\right.$,

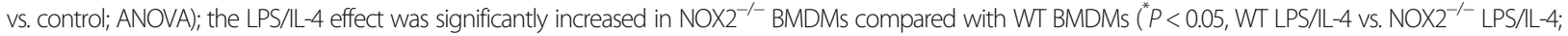
ANOVA). SOCS3 and IL-10 expression levels were significantly increased in the presence of alL-10 $\left({ }^{\wedge} P<0.01,{ }^{\wedge \wedge \wedge} P<0.001\right.$, NOX2 $2^{-/-}$LPS/IL-4 VS. $\mathrm{NOX}^{-1-}$ LPS/IL-4 + alL-10; ${ }^{\# \# \#} P<0.001$, WT LPS/IL-4 vs. WT LPS/IL-4 + alL-10; ANOVA). All data are expressed as means $( \pm$ SEM, $n=6)$

and 7 days post-injury (Fig. 6a). IL-10 mRNA expression was significantly increased in WT and $\mathrm{NOX}^{-/-} \mathrm{TBI}$ mice when compared to sham levels at all time points examined. Notably, there was a significant increase in
IL-10 mRNA expression in NOX2 $2^{-1-}$ TBI mice at $72 \mathrm{~h}$ and 7 days when compared to WT TBI mice $(P<0.05$; [72 h]; $P<0.01$ [7 days]; ANOVA; Fig. 6a). To identify the cellular source of IL-10, we combined IL-10 mRNA 


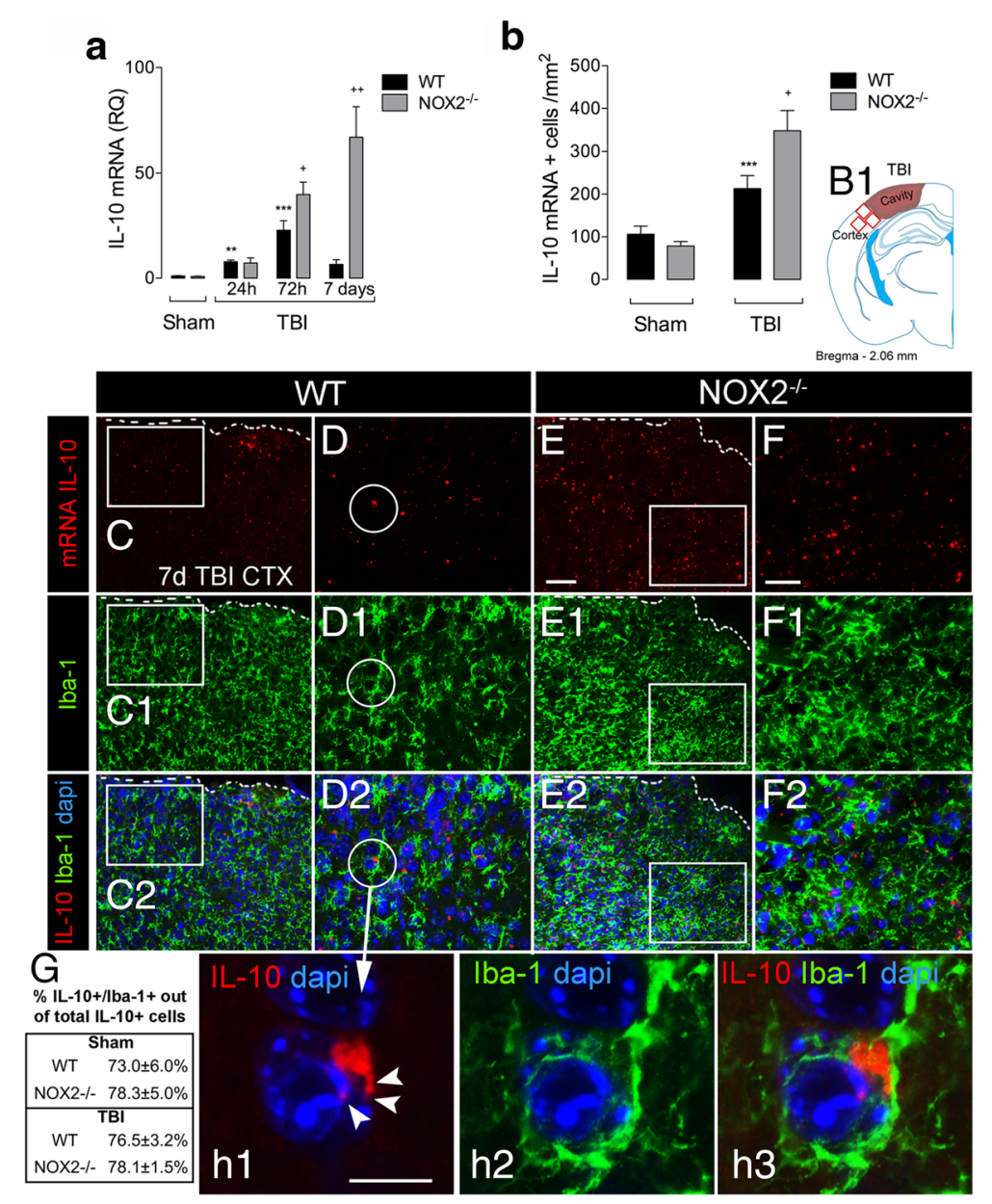

Fig. $6 \mathrm{IL}-10$ is increased in the cortex of NOX2 $2^{-/-}$TBI mice. (A) IL-10 mRNA expression was assessed in the ipsilateral cortex of WT and NOX2 $2^{-/-}$sham and TBI mice at 24 , and $72 \mathrm{~h}$ and 7 days post-injury. TBI increased IL-10 mRNA at all time points $\left({ }^{* *} P<0.01,[24 \mathrm{~h}],{ }^{* * * *} \mathrm{P}<0.001\right.$ [72 h], Vs. sham; ANOVA). IL-10 expression was significantly increased in NOX2 ${ }^{-/-}$TBI mice at $72 \mathrm{~h}$ and 7 days $\left({ }^{+} P<0.05,{ }^{++} \backslash P<0.01\right.$, WT TBI vs. NOX2 $2^{-/-}$TBl; ANOVA). All data are expressed as means ( \pm SEM, $n=5$ ). (B) Quantification of IL-10 mRNA positive cells in the ipsilateral cortex of WT and NOX2 ${ }^{-1-}$ sham and TBI mice at 7 days post-injury. TBI increased IL-10 expression when compared to sham $\left({ }^{* * *} P<0.001\right.$; ANOVA), and this effect was significantly greater in NOX2 $2^{-1-}$ TBI mice $\left({ }^{+} P<0.05, W T\right.$ TBI vs. NOX2 ${ }^{-/-}$TBl; ANOVA). All data are expressed as means $( \pm \mathrm{SEM}, n=5)$. (B1) Schematic representation of the injured coronal brain section, the red squares indicate the fields that were examined in this study. $(\mathbf{C}-\mathbf{H})$ Representative images from the ipsilateral cortex of WT (C-D2) and NOX2 $2^{-1-}$ (E-F2) TBI mice at 7 days post-injury. Images show reduced IL-10 positive cells (red) in the injured cortex of WT TBI (C, inset in $\mathbf{D})$ compared to NOX2 $2^{-1-}$ TBI mice (E, inset in $\mathbf{F}$ ). IL-10 was predominantly expressed in microglia/macrophages detected by immunohistochemistry (Iba-1, green) and nuclei (dapi, blue) in both groups; WT (C1-C2, insets in D-D2) and in NOX2 ${ }^{-1-}$ TBI mice (E1-E2, insets in F2-F3). A white striped line delineates the cavity and the perilesional cortex (C-C2 and $\mathbf{E}-\mathbf{E} 2$ ). (G) Table illustrating quantification analysis of \%lL-10/lba-1+ cells in the ipsilateral cortex of WT and NOX2 ${ }^{-/-}$sham and TBI mice. (H) High magnification (inset circle in D-D2) of a representative image for IL-10 positive (h1) microglia/ macrophage (h2-h3 Iba-1 in green, dapi in blue) showing cytoplasmic and perinuclear IL-10 mRNA expression with puncta spike that represents a single IL-10 mRNA transcript (white arrowheads in h1). Scale bars $75 \mu \mathrm{m}$ for C-C2 and E-E2; F2; $50 \mu \mathrm{m}$ D-D2, F-F2; and $20 \mu \mathrm{m}$ for h1-h3

FISH with immunohistochemical markers for macrophages/microglia in the ipsilateral cortex of WT and $\mathrm{NOX}^{-1-}$ sham and TBI mice at 7 days post-injury. There was a significant increase in IL-10-positive cells in the cortex of $\mathrm{NOX} 2^{-1-} \mathrm{TBI}$ mice when compared to WT TBI mice $(P<0.05$; ANOVA; Fig. $6 \mathrm{~b})$, and approximately $78 \%$ of the IL-10-positive cells were Iba-1-positive macrophages/microglia (representative images Fig. 6c-h). Together, these data indicate that NOX2 deficiency results in a significant increase in IL-10 expression in macrophages/microglia during the acute-subacute phase following TBI.

Increased anti-inflammatory marker expression in the cortex of NOX2 $2^{-1-}$ TBI mice is associated with enhanced IL-10/STAT3 signaling

IL-10 expression was enhanced in $\mathrm{NOX}^{-/-} \mathrm{TBI}$ mice compared to WT TBI mice; therefore, we next assessed STAT3 and STAT6 signaling pathways and antiinflammatory marker expression at $72 \mathrm{~h}$ post-injury. TBI 
significantly increased the expression of pSTAT3 in the ipsilateral cortex of both WT and $\mathrm{NOX2}^{-1-}$ mice $(P<0.001$; ANOVA; Fig. $7 b)$. Notably, STAT3 activation was robustly and significantly increased in $\mathrm{NOX}^{-1-} \mathrm{TBI}$ mice when compared to WT TBI mice $(P<0.05$; ANOVA; Fig. 7b). STAT6 activation was increased in WT and NOX2 ${ }^{-1-}$ TBI mice $(P<0.01$; ANOVA; Fig. $7 \mathrm{c})$; however, there was no genotype-related change in pSTAT6 expression. In addition, TBI resulted in a significant increase in the cortical expression of IL-4R $\alpha$ $(P<0.001 ; \quad$ ANOVA; $\quad$ Fig. $7 d), \quad$ SOCS3 $\quad(P<0.001$; ANOVA; Fig. 7e), TGF $\beta(P<0.001$; ANOVA; Fig. $7 \mathrm{f})$, SHIP1 $(P<0.001$; ANOVA; Fig. $7 g), \operatorname{Arg} 1 \quad(P<0.001$; ANOVA; Fig. 7h), and YM1 $(P<0.01$; ANOVA; Fig. $7 \mathrm{i})$ mRNA in WT and NOX2 ${ }^{-1-}$ TBI mice. The injury effect on inflammatory markers downstream of IL-10 was significantly increased in the ipsilateral cortex of $\mathrm{NOX} 2^{-/-}$ TBI mice when compared to WT TBI mice $(P<0.05$
[IL-4Ro; SOCS3; Arg1; YM1]; $P<0.001$ [SHIP1; TGF $\beta$; ANOVA; Fig. 7d-i; also Additional file 1: Table S1).

\section{IL-10 promotes an anti-inflammatory environment in $\mathrm{NOX2}^{-/-}$mice following TBI}

Finally, to determine if IL-10 promotes the antiinflammatory response in NOX2 $2^{-1-}$ TBI mice, we blocked IL-10 in the brain using the IL-10 neutralization strategy that we employed in prior BMDM studies. To achieve this, WT mice and $\mathrm{NOX}^{-/-}$TBI mice had an i.c.v. osmotic pump implanted on the contralateral side to infuse neutralizing $\alpha \mathrm{IL}-10(1 \mathrm{mg} / \mathrm{ml})$ or equal concentration isotype control $\alpha \operatorname{IgG}(\alpha \operatorname{IgG} 2 b \mathrm{~K})$ into the lateral ventricle for $72 \mathrm{~h}$, after which ipsilateral cortex tissue was collected to assess anti-inflammatory markers (Fig. 8a). TBI increased the expression of IL-4R $\alpha(P<0.001$; ANOVA; Fig. $8 \mathrm{~b})$, SOCS3 $(P<0.001$; ANOVA; Fig. $8 \mathrm{c})$, TGF $\beta(P<0.001$; ANOVA; Fig. 8d), YM1 $(P<0.001$; ANOVA; Fig. 8e), and Arg1

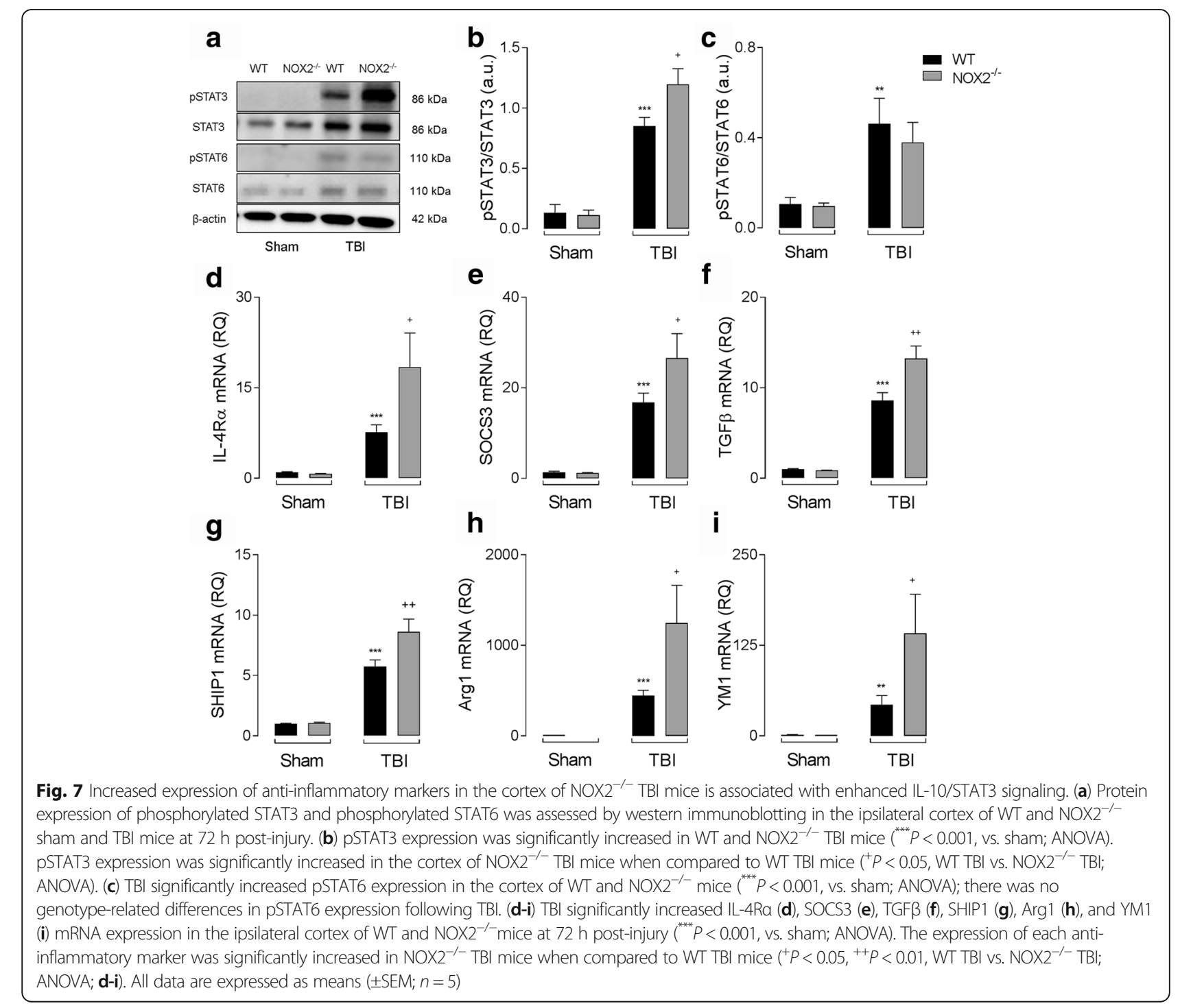



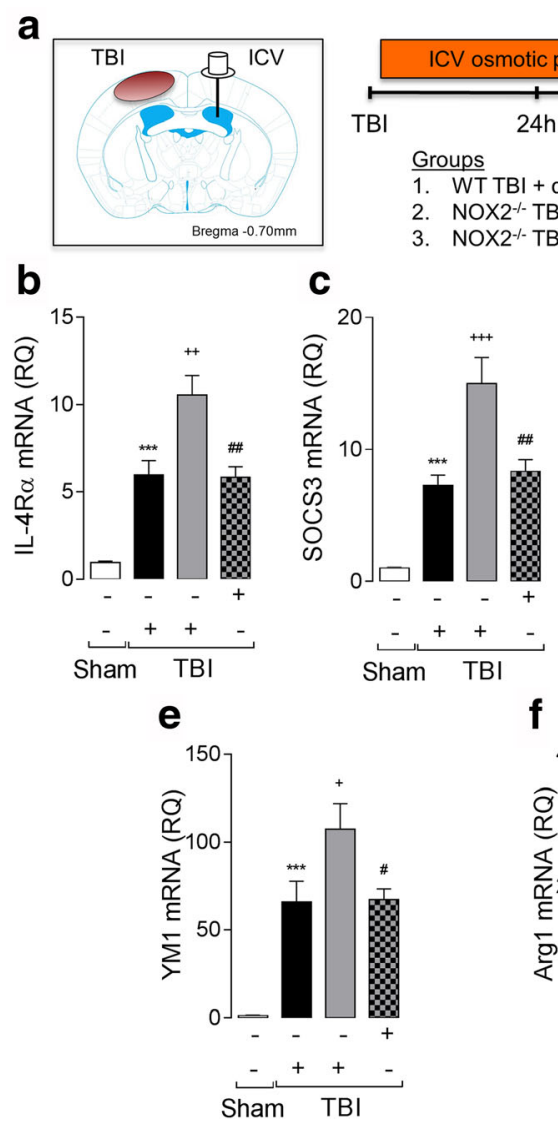

ICV osmotic pump infusion (Groups 1-3)

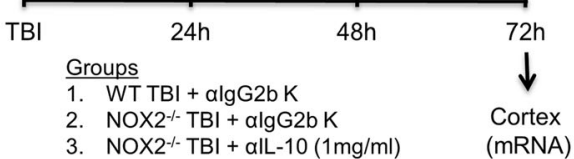

d
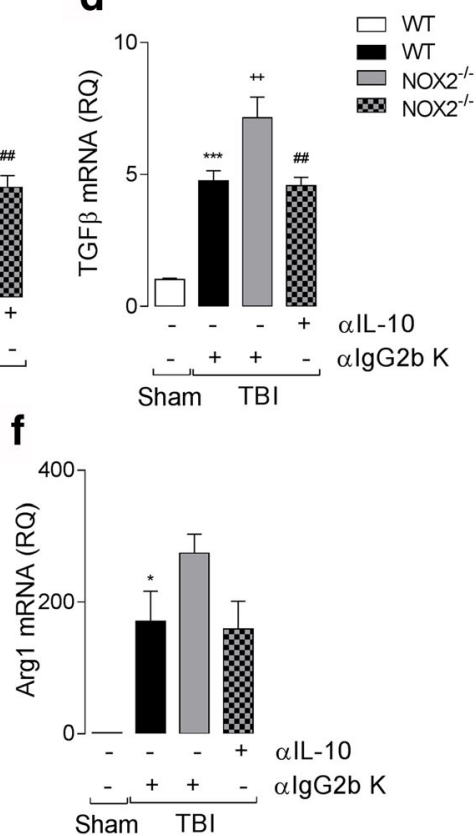

Fig. $8 \mathrm{LL}-10$ promotes an anti-inflammatory environment in cortex of NOX2-/- TBI mice. (a) Neutralizing anti-IL-10 (alL-10; 1 mg/ml, i.c.v.) or control algG2b K was administered to WT or NOX2 $2^{-1-}$ TBI mice for $72 \mathrm{~h}$, and ipsilateral cortex tissue was collected for mRNA analysis. (b-f) TBI increased IL-4Ra (b), SOCS3 (c), TGF $(\mathbf{d})$, YM1 (e), and Arg1 (f) mRNA expression in the ipsilateral cortex of WT and NOX2 ${ }^{-/}{ }^{-}$mice $\left(^{* * *} P<0.001\right.$, vs. sham; ANOVA). TBI effect on anti-inflammatory marker expression was significantly increased in NOX2 ${ }^{-/-} \mathrm{TBI}$ mice when compared to WT TBI mice $\left({ }^{+} P<0.05,{ }^{++} P<0.01,{ }^{+++} p<0.001\right.$, WT algG2b K TBI vs. NOX2 ${ }^{-/-}$algG2b K TBl; ANOVA). alL-10 treatment significantly reduced the expression of all anti-inflammatory markers in NOX2 $2^{-/}$TBI mice $\left({ }^{\#} P<0.05,{ }^{\# \#} P<0.01, \mathrm{NOX} 2^{-/-}\right.$algG2b K TBI vs. NOX2 ${ }^{-/-}$alL-10 TBl; ANOVA). All data are expressed as means $( \pm \mathrm{SEM} ; n=6)$

$(P<0.05$; ANOVA; Fig. 8f $)$ mRNA in all TBI mice compared to WT sham mice. As predicted, the effect of TBI on IL-4R $\alpha$, SOCS3, TGF $\beta$, and YM1 was significantly increased in $\mathrm{NOX}^{-/}$TBI mice compared to WT TBI mice $(P<0.05$ [YM1]; $P<0.01$ [IL-4R $\alpha$ TGF $\beta$ ]; $P<0.001$ [SOCS3]; ANOVA; Fig. 8b-e). Notably, the NOX2 ${ }^{-/}$-dependent upregulation of anti-inflammatory markers after TBI was significantly reduced when IL-10 was neutralized using $\alpha \mathrm{IL}-10$, such that the $\alpha \mathrm{IL}-10$-treated NOX2 $-/-$ TBI group returned to $\alpha$ IgG-treated WT TBI levels $(P<0.05 \quad$ [YM1]; $\quad P<0.01 \quad[\mathrm{IL}-4 \mathrm{R} \alpha ; \quad$ SOCS3; TGF $]$; ANOVA; Fig. 8b-e).

\section{Discussion}

Redox signaling regulates macrophage responses during inflammation [16], and NOX2/ROS are implicated in secondary neuroinflammatory cascades in ischemic brain injury $[35,36]$, spinal cord injury (SCI) $[37,38]$, and TBI $[21,23$, 26]. NOX2 is highly expressed in macrophage/microglia up to 12 months after moderate-to-severe TBI [21], and inhibition of NOX2 is neuroprotective and reduces inflammatory-mediated neuronal cell death after TBI [2326]. We recently reported that NOX2 inhibition also enhances anti-inflammatory activation of macrophage/microglia following TBI [24]. Thus, NOX2 may act as a critical switch between pro- and anti-inflammatory phenotypes after TBI. Similar findings in SCI [37, 39] and stroke [40] models indicate that this may be a common cellular mechanism that regulates CNS injury responses. Therefore, the goal of the present study was to investigate signaling pathways that regulate NOX2-dependent phenotypic changes in macrophages and relate these signaling events back to TBI. To this end, we developed an in vitro model of macrophage activation that mimics characteristic features of the mixed pro- and anti-inflammatory phenotypes observed in the TBI brain [12-14], and used it to identify IL-10-STAT3 signaling as a key mechanism by which NOX2 inhibition regulates macrophage activation states after TBI. 
Whereas many previous studies have demonstrated that macrophages/microglia in vitro can be stimulated to adopt specific phenotypes, it appears less likely that such defined phenotypes exist in vivo [9]. We, and others, have demonstrated that following brain trauma macrophages/microglia adopt mixed phenotypes and express both pro and anti-inflammatory activation markers [12-14]. Here, we demonstrated that similar to the activation profile in the TBI brain, macrophages stimulated with combined LPS/IL-4, but not LPS or IL-4 alone, upregulated mixed phenotypic markers associated with both pro- and anti-inflammatory activation states. When we compared the responses of WT and NOX2 $2^{-/-}$ BMDMs to the mixed LPS/IL-4 stimulation model, we found that NOX2 deficiency was associated with decreased production of pro-inflammatory mediators. This activation profile is consistent with prior studies in $\mathrm{NOX}^{-/-}$microglia exposed to LPS challenge $[27,41]$. There was also a significant increase in anti-inflammatory activation markers in $\mathrm{NOX}^{-/-}$BMDMs. These findings are significant because there are increased numbers of anti-inflammatory microglia/macrophages in the injured cortex of NOX2 $2^{-/-}$TBI mice [24]. Of note, it was not possible to promote this NOX2-dependent phenotype shift when BMDMs were treated with only pro-inflammatory stimuli (LPS or IFN $\gamma$ alone; data not shown) or antiinflammatory stimuli (IL-4 alone; data not shown), highlighting the importance of mixed environmental stimuli (combined LPS/IL-4) for NOX2-dependent macrophage phenotype responses. In the context of TBI, it is intriguing to speculate on the significance of these stimulus-driven effects such that they highlight intrinsic tension between activation pathways. Similar to responses in wound healing macrophages [42], coincident pro- and anti-inflammatory activation may be an attempt to fine-tune the injury response in which the two pathways support each other to facilitate neuroprotection and immune resolution that may be important for neurorestoration after TBI, thereby revealing their complementary nature. However, complete absence of a molecular signaling cascade (activation by only pro(LPS) or anti-inflammatory (IL-4) stimuli in BMDMs) or the loss of molecular signaling over time (dominant NOX2 response after TBI [12]) may significantly impact the functional effects of the opposite microglial/macrophage activation state.

We investigated the impact of NOX2 deficiency on signaling cascades involved in regulating macrophage phenotypes, with particular emphasis on mechanisms that drive anti-inflammatory activation that may be relevant for neuroprotection and neurorestoration following TBI. The role of STAT1 in the induction of the proinflammatory phenotype is well-established [43], and STAT1 is required for LPS-induced gene expression in macrophages [5]. In our model, the mixed LPS/IL-4 stimulus significantly increased pSTAT1 expression in WT and NOX2 $2^{-/-}$BMDMs, but STAT1 activation was significantly reduced in $\mathrm{NOX}_{2}^{-/-}$BMDMs. This is in agreement with a previous report where NOX deficient macrophages incubated in the presence of IFN $\gamma / \mathrm{LPS}$ exhibited decreased STAT1 activation [44]. In addition, previous work demonstrated that LPS-induced IFN $\beta$ is required for STAT1 activation [45], and this may be a mechanism that explains reduced pSTAT1 and decreased pro-inflammatory marker expression in NOX2 -I- BMDMs.

IL-4 stimulation increases the expression of markers associated with the anti-inflammatory phenotype, and STAT6 activation appears to be essential for driving these changes [6]. Our recent study suggested that the enhanced anti-inflammatory environment in the cortex of $\mathrm{NOX}^{-1-}$ TBI mice is mediated, at least in part, by IL-4R $\alpha$-positive infiltrating macrophages [24]. Here, we demonstrated that LPS/IL-4 treatment increased antiinflammatory markers in $\mathrm{NOX}^{-/-} \mathrm{BMDMs}$ when compared to WT BMDMs. Unexpectedly, we observed no genotype-related change in STAT6 activation, which conflicts with a prior report showing that pSTAT6 expression is enhanced in $\mathrm{NOX}^{-/-}$BMDMs [44]. Such discrepancies may be due to the different activation stimuli employed in each study. Nevertheless, the results from the current study suggest that enhanced anti-inflammatory changes in $\mathrm{NOX}^{-/-}$BMDMs are not mediated by increased STAT6 signaling.

Having established that anti-inflammatory responses in $\mathrm{NOX}^{-/-}$BMDMs were not due to enhanced STAT6 activation, we then identified an alternative pathway that could mediate these effects. IL-10 is a pleiotropic immunoregulatory cytokine that plays a critical role in controlling inflammation and immune responses [46]. IL-10 regulates TLR signaling [47], and IL-10 deficient mice exhibit exaggerated responses to LPS [48]. IL-10 can also enhance antiinflammatory activation induced by IL-4 [7]. Here, we show that LPS/IL-4 stimulation enhances IL-10 release, an effect which is robustly increased in NOX2 -I- BMDMs. These data are consistent with a recent report showing that ROS inhibition using a mitochondrial complex 1 inhibitor decreases LPS-induced activation of macrophages and increases IL-10 production [49]. IL-10 mediates its effects through activation of STAT3 [50], and in the current study we found that STAT3 signaling was significantly increased in $\mathrm{NOX2}^{-/-}$ BMDMs. Stimulation of macrophages with IL-10 leads to the upregulation of several genes, including IL-4R $\alpha$ and SOCS3 [34], and in response to LPS/IL-4, mRNA expression of both markers was significantly increased in NOX2 -/- compared to WT BMDMs. 
To investigate whether IL-10 plays a critical role in the NOX2-dependent phenotype switch, WT and NOX2 $2^{-/-}$ BMDMs were stimulated with LPS/IL-4 in the presence or absence of a neutralizing $\alpha \mathrm{IL}-10$ antibody. Increased pSTAT3 expression in $\mathrm{NOX}^{-1-}$ BMDMs was attenuated in the presence of $\alpha \mathrm{IL}-10$, demonstrating that the genotype-related effect on STAT3 activation was mediated by IL-10. IL-4-stimulated macrophage activation can be synergized by IL-10 [7], and when IL-10 was neutralized, there was a significant reduction in IL-4R $\alpha$ and Arg1. Interestingly, when IL-10 was neutralized, the LPS/IL-4 effects on SOCS3 and IL-10 were further increased; significantly, the NOX2 genotype-related effects were abolished with $\alpha \mathrm{IL}-10$ treatment. These observations are consistent with the fact that TLR4 activation induces SOCS3 and IL-10 expression [32, 51]; by removing the inhibitory effect of IL-10, LPS-induced changes are increased. Together, these data demonstrate that in the absence of NOX2, there is increased IL-10/ STAT3 signaling, which in turn results in enhanced antiinflammatory macrophage activation.

Clinically, IL-10 is upregulated during the acute phase after moderate-to-severe TBI $[52,53]$, and several pre- clinical studies report neuroprotective effects of IL-10 treatment in acute brain injury that can be attributed to its anti-inflammatory activities [54-56]. IL-10 treatment produces strong neuroprotective effects in LPS-induced dopaminergic neurotoxicity models [57], and these effects are mediated by inhibition of microglial activation and NOX2-dependent ROS in neuron-glia co-cultures. In the present study, we demonstrated that IL-10 expression was increased in the ipsilateral cortex of WT and $\mathrm{NOX}^{-1-} \mathrm{TBI}$ mice, and that this effect was significantly greater in $\mathrm{NOX}^{-/-}$animals. Importantly, our in situ analysis showed that IL-10 expression was robustly upregulated in approximately $78 \%$ of macrophages/ microglia within the injured cortex of $\mathrm{NOX}^{-/-} \mathrm{TBI}$ mice. We previously identified the peak of macrophage infiltration after TBI to be at $72 \mathrm{~h}$ post-injury [12], and this is the same time point at which IL-10 expression is maximal in WT mice. There was also increased pSTAT3 expression, but no change in STAT6 activation, in TBI $\mathrm{NOX}^{-/-}$mice at $72 \mathrm{~h}$ post-injury, suggesting that the changes observed were as a result of increased STAT3 activity. STAT3 activation has been described in secondary injury responses following TBI and SCI [58-60].

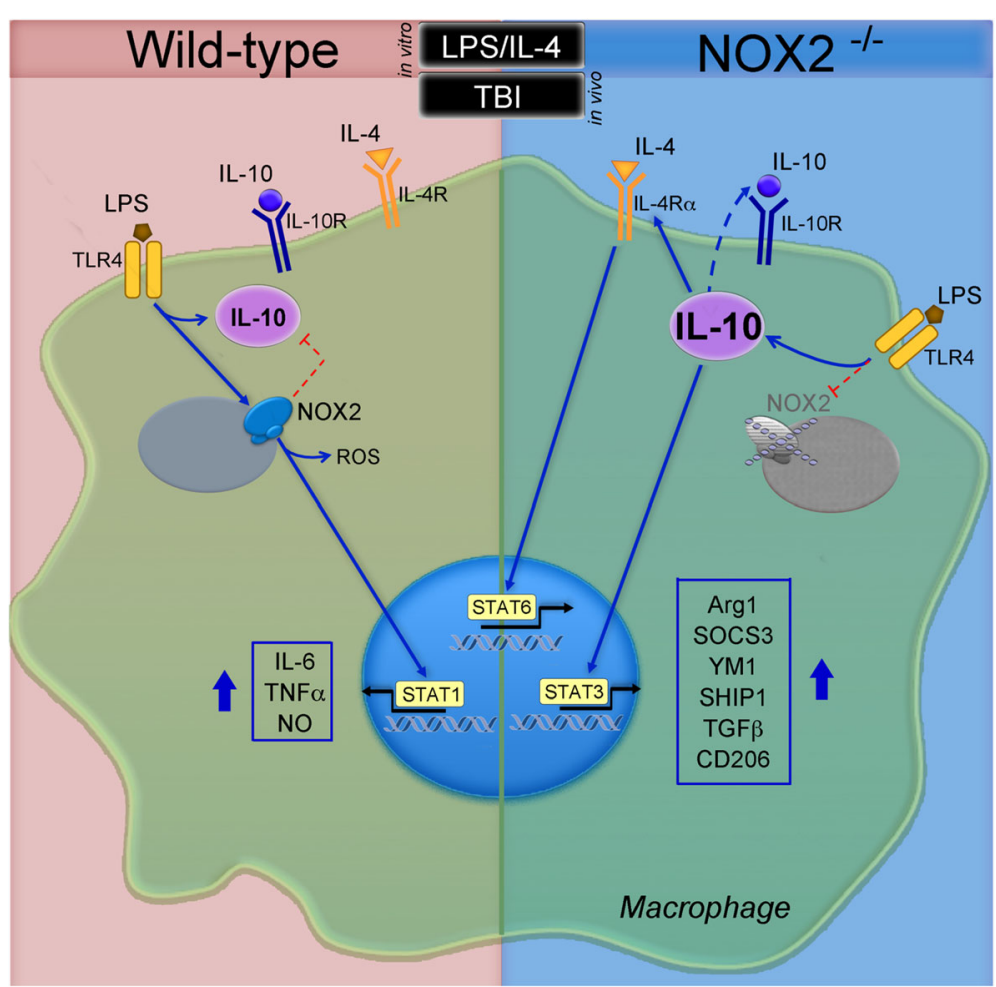

Fig. 9 Schematic of NOX2 regulation of macrophage activation. Activation of TLR4 initiates a signaling cascade that leads to activation of NOX2 and increased ROS production; this in turn induces the activation of STAT1, which leads to an increase in the production of pro-inflammatory mediators. TLR4 signaling also induces the production of IL-10, which acts in autocrine fashion to regulate TLR4-induced changes. In NOX $2^{-/}$ cells, TLR4-induced changes are significantly different; NOX2-deficiency is associated with a decrease in pro-inflammatory markers and significantly greater IL-10 production. This increase in IL-10 leads to a robust increase in STAT3 signaling, which in conjunction with STAT6 activation, leads to increased expression of anti-inflammatory markers 
Although some studies have suggested a beneficial role [59], others have reported that STAT3 activation is detrimental [61]. These conflicting reports highlight the complexity of STAT3 activation in secondary injury and differential STAT3 cellular responses during acute CNS injury (STAT3 effects in glia vs. neurons). Here, we clearly demonstrate that NOX2 deficiency resulted in increased STAT3 activation in macrophages (BMDMs), and that a similar STAT3 activation profile was observed in the injured cortex of $\mathrm{NOX} 2^{-1-} \mathrm{TBI}$ mice. In addition to enhanced STAT3 signaling, several genes downstream of IL-10 (IL-4R $\alpha$, TGF $\beta$, SOCS3, SHIP1) and other anti-inflammatory genes (Arg1 and YM1) were robustly upregulated in the injured cortex of $\mathrm{NOX}^{-/-} \mathrm{TBI}$ mice. Therefore, despite no differences in IL-4-mediated signaling, our data indicate that the anti-inflammatory response in $\mathrm{NOX}^{-/-} \mathrm{TBI}$ mice may be as a result of enhanced IL-10/STAT3 signaling.

Finally, to address whether IL-10 is essential for the enhanced anti-inflammatory environment in $\mathrm{NOX}^{-/-} \mathrm{TBI}$ mice, we used a neutralizing antibody to inhibit IL-10 within the CNS as previously described [29]. After IL-10 neutralization, the TBI-induced increase in expression of anti-inflammatory markers in $\mathrm{NOX}^{-1-} \mathrm{TBI}$ mice was significantly reduced compared to expression levels in IgG control NOX2 $2^{-/}$TBI mice. These results indicate that IL10 is a major contributor to the anti-inflammatory environment in the injured cortex of NOX2 $2^{-1-}$ TBI mice. When combined with our recent report [24], these studies indicate that NOX2 inhibition in macrophages switches them towards an anti-inflammatory phenotype through an IL-10/ STAT3 dependent pathway, which may contribute to the neuroprotective effects that result in long-term neurological recovery after TBI. Interestingly, following SCI infiltrating monocyte-derived macrophages that display an immunoregulatory phenotype with high IL-10 expression play a critical role in controlling the inflammatory response within the lesion microenvironment, which in turn is associated with improved motor function recovery [62]. Furthermore, adoptive transfer of anti-inflammatory macrophages that infiltrate the lesion site after SCI where they produce high levels of IL-10 and IL-13 to promote axon remyelination and preserve neuronal function [63].

\section{Conclusions}

These studies demonstrate that in response to mixed inflammatory stimuli $\mathrm{BMDMs}$ from $\mathrm{NOX}^{-/-}$mice exhibit decreased pro-inflammatory activation, and a concomitant increase in anti-inflammatory activation, and that this switch is IL-10/STAT3-dependent (Fig. 9). Furthermore, our in vivo TBI studies indicate that NOX2 deficiency drives an anti-inflammatory response in the injured cortex that is mediated by IL-10. Overall, these findings indicate that interventions that inhibit NOX2 activity in macrophages/microglia may improve outcomes following TBI not only by limiting pro-inflammatory and neurotoxic responses, but also by enhancing IL-10-mediated responses that are neuroprotective.

\section{Additional file}

Additional file 1: Table S1. Average $C T$ values of genes measured by qPCR in the cortex of SHAM and TBI (72 h). (TIF $126 \mathrm{~kb}$ )

\section{Abbreviations}

ANOVA: Analysis of variance; Arg1: Arginase-1; BMDM: Bone marrow-derived macrophage; CCl: Controlled cortical impact; CD206: Mannose receptor; FISH: Fluorescent In Situ Hybridization; i.c.V.: Intracerebroventricular; IFNү: Interferon- $\gamma$; IGF-1: Insulin-stimulating growth factor-1; IL-10: Interleukin-10; IL-4: Interleukin-4; IL-6: Interleukin-6; LPS: Lipopolysaccharide; NO: Nitric oxide; NOS2: Nitric oxide synthase; NOX2: NADPH Oxidase; NOX2 $2^{-/-}$: NOX2 deficient; ROS: Reactive oxygen species; SCl: Spinal cord injury; SOCS: Suppressor of cytokine signaling; STAT: Signal transducer activator of transcription; TBI: Traumatic brain injury; TLR: Toll-like receptors; TNF: Tumor necrosis factor

\section{Funding}

This work was supported by NIH grant R01NS082308 (D.J. Loane), R01NS037313 (A.I. Faden), R01 NS067417 (M.P. Burns), and The National Institute on Aging (NIA) Claude D. Pepper Older Americans Independence Center P30-AG028747 (D.J. Loane).

\section{Availability of data and materials}

Data supporting the Conclusions of this article are presented in the manuscript.

\section{Authors' contributions}

JPB designed the experiments and carried out all in vitro and in vivo experiments. RJH and DJL carried out surgical procedures. SV and MPB designed and carried out FISH/IHC analysis. AK analyzed the data. DJL and JPB wrote the manuscript. BAS and AIF revised the manuscript. DJL conceived and coordinated the study. All authors reviewed the results and approved the final version of the manuscript.

\section{Competing interests}

The other authors declare that they have no competing interests

Consent for publication

Not applicable.

\section{Ethics approval}

All procedures and animal experiments were carried out in accordance with protocols approved by the Animal Care and Use Committee at the University of Maryland.

\section{Publisher's Note}

Springer Nature remains neutral with regard to jurisdictional claims in published maps and institutional affiliations.

\section{Author details}

${ }^{1}$ Department of Anesthesiology and Shock, Trauma and Anesthesiology Research (STAR) Center, University of Maryland School of Medicine, 655 West Baltimore Street, \#6-011, Baltimore, MD 21201, USA. ${ }^{2}$ Laboratory for Brain Injury and Dementia, Department of Neuroscience, Georgetown University, Washington, DC, USA.

Received: 25 January 2017 Accepted: 16 March 2017

Published online: 24 March 2017

\section{References}

1. Sica A, Mantovani A. Macrophage plasticity and polarization: in vivo veritas. J Clin Invest. 2012;122:787-95. 
2. Loane DJ, Kumar A. Microglia in the TBI brain: the good, the bad, and the dysregulated. Exp Neurol. 2016;275(Pt 3):316-27.

3. Murray PJ, Allen JE, Biswas SK, Fisher EA, Gilroy DW, Goerdt S, Gordon S, Hamilton $J A$, Ivashkiv LB, Lawrence T, et al. Macrophage activation and polarization: nomenclature and experimental guidelines. Immunity. 2014;41:14-20.

4. Gordon S, Martinez FO. Alternative activation of macrophages: mechanism and functions. Immunity. 2010;32:593-604.

5. Ohmori Y, Hamilton TA. Requirement for STAT1 in LPS-induced gene expression in macrophages. J Leukoc Biol. 2001;69:598-604.

6. Takeda K, Tanaka T, Shi W, Matsumoto M, Minami M, Kashiwamura S, Nakanishi K, Yoshida N, Kishimoto T, Akira S. Essential role of Stat6 in IL-4 signalling. Nature. 1996;380:627-30.

7. Makita N, Hizukuri Y, Yamashiro K, Murakawa M, Hayashi Y. IL-10 enhances the phenotype of $\mathrm{M} 2$ macrophages induced by $\mathrm{IL}-4$ and confers the ability to increase eosinophil migration. Int Immunol. 2015;27:131-41.

8. Barrett JP, Minogue AM, Falvey A, Lynch MA. Involvement of IGF-1 and Akt in M1/M2 activation state in bone marrow-derived macrophages. Exp Cell Res. 2015;335:258-68.

9. Gordon S, Pluddemann A, Martinez Estrada F. Macrophage heterogeneity in tissues: phenotypic diversity and functions. Immunol Rev. 2014;262:36-55.

10. Ransohoff RM. A polarizing question: do M1 and M2 microglia exist? Nat Neurosci. 2016;19:987-91.

11. Ginhoux F, Schultze JL, Murray PJ, Ochando J, Biswas SK. New insights into the multidimensional concept of macrophage ontogeny, activation and function. Nat Immunol. 2016;17:34-40.

12. Kumar A, Alvarez-Croda DM, Stoica BA, Faden Al, Loane DJ. Microglial/ Macrophage Polarization Dynamics following Traumatic Brain Injury. J Neurotrauma. 2016;33:1732-1750

13. Morganti JM, Riparip LK, Rosi S. Call Off the Dog(ma): M1/M2 Polarization Is Concurrent following Traumatic Brain Injury. PLoS One. 2016;11:e0148001.

14. Kim CC, Nakamura MC, Hsieh CL. Brain trauma elicits non-canonical macrophage activation states. J Neuroinflammation. 2016;13:117.

15. Bedard K, Krause KH. The NOX family of ROS-generating NADPH oxidases: physiology and pathophysiology. Physiol Rev. 2007;87:245-313.

16. Brune B, Dehne N, Grossmann N, Jung M, Namgaladze D, Schmid T, von Knethen A, Weigert A. Redox control of inflammation in macrophages, Antioxid Redox Signal. 2013;19:595-637.

17. Shimohama S, Tanino H, Kawakami N, Okamura N, Kodama H, Yamaguchi T, Hayakawa T, Nunomura A, Chiba S, Perny G, et al. Activation of NADPH oxidase in Alzheimer's disease brains. Biochem Biophys Res Commun. 2000;273:5-9.

18. Wu DC, Teismann P, Tieu K, Vila M, Jackson-Lewis V, Ischiropoulos H, Przedborski S. NADPH oxidase mediates oxidative stress in the 1-methyl-4phenyl-1,2,3,6-tetrahydropyridine model of Parkinson's disease. Proc Natl Acad Sci U S A. 2003;100:6145-50.

19. Fischer MT, Sharma R, Lim JL, Haider L, Frischer JM, Drexhage J, Mahad D, Bradl M, van Horssen J, Lassmann H. NADPH oxidase expression in active multiple sclerosis lesions in relation to oxidative tissue damage and mitochondrial injury. Brain. 2012;135:886-99

20. Byrnes KR, Loane DJ, Stoica BA, Zhang J, Faden Al. Delayed mGluR5 activation limits neuroinflammation and neurodegeneration after traumatic brain injury. J Neuroinflammation. 2012;9:43.

21. Loane DJ, Kumar A, Stoica BA, Cabatbat R, Faden Al. Progressive neurodegeneration after experimental brain trauma: association with chronic microglial activation. J Neuropathol Exp Neurol. 2014;73:14-29.

22. Kumar A, Stoica BA, Sabirzhanov B, Burns MP, Faden Al, Loane DJ. Traumatic brain injury in aged animals increases lesion size and chronically alters microglial/macrophage classical and alternative activation states. Neurobiol Aging. 2013;34:1397-411.

23. Dohi K, Ohtaki H, Nakamachi T, Yofu S, Satoh K, Miyamoto K, Song D, Tsunawaki S, Shioda S, Aruga T. Gp91 phox (NOX2) in classically activated microglia exacerbates traumatic brain injury. J Neuroinflammation. 2010:7:41.

24. Kumar A, Barrett JP, Alvarez-Croda DM, Stoica BA, Faden Al, Loane DJ. NOX2 drives M1-like microglial/macrophage activation and neurodegeneration following experimental traumatic brain injury. Brain Behav Immun. 2016;58:291-309.

25. Loane DJ, Stoica BA, Byrnes KR, Jeong W, Faden Al. Activation of mGluR5 and inhibition of NADPH oxidase improves functional recovery after traumatic brain injury. J Neurotrauma. 2013;30:403-12.

26. Zhang QG, Laird MD, Han D, Nguyen K, Scott E, Dong Y, Dhandapani KM Brann DW. Critical role of NADPH oxidase in neuronal oxidative damage and microglia activation following traumatic brain injury. PLoS One. 2012;7:e34504.
27. Choi SH, Aid S, Kim HW, Jackson SH, Bosetti F. Inhibition of NADPH oxidase promotes alternative and anti-inflammatory microglial activation during neuroinflammation. J Neurochem. 2012;120:292-301.

28. Loane DJ, Pocivavsek A, Moussa CE, Thompson R, Matsuoka Y, Faden Al, Rebeck GW, Burns MP. Amyloid precursor protein secretases as therapeutic targets for traumatic brain injury. Nat Med. 2009:15:377-9.

29. Ishii H, Tanabe S, Ueno M, Kubo T, Kayama H, Serada S, Fujimoto M, Takeda K, Naka T, Yamashita T. ifn-gamma-dependent secretion of IL-10 from Th1 cells and microglia/macrophages contributes to functional recovery after spinal cord injury. Cell Death Dis. 2013;4:e710.

30. Villapol S, Balarezo MG, Affram K, Saavedra JM, Symes AJ. Neurorestoration after traumatic brain injury through angiotensin II receptor blockage. Brain. 2015;138: 3299-315

31. Mosser DM, Edwards JP. Exploring the full spectrum of macrophage activation. Nat Rev Immunol. 2008:8:958-69.

32. Chhor V, Le Charpentier T, Lebon S, Ore MV, Celador IL, Josserand J, Degos V, Jacotot E, Hagberg H, Savman K, et al. Characterization of phenotype markers and neuronotoxic potential of polarised primary microglia in vitro. Brain Behav Immun. 2013:32:70-85.

33. Heller NM, Qi X, Junttila IS, Shirey KA, Vogel SN, Paul WE, Keegan AD. Type I IL-4Rs selectively activate IRS-2 to induce target gene expression in macrophages. Sci Signal. 2008; 1:ra17.

34. Gordon S. Alternative activation of macrophages. Nat Rev Immunol. 2003;3: 23-35.

35. Chen H, Kim GS, Okami N, Narasimhan P, Chan PH. NADPH oxidase is involved in post-ischemic brain inflammation. Neurobiol Dis. 2011;42:341-8.

36. Chen H, Song YS, Chan PH. Inhibition of NADPH oxidase is neuroprotective after ischemia-reperfusion. J Cereb Blood Flow Metab. 2009;29:1262-72.

37. Khayrullina G, Bermudez S, Byrnes KR. Inhibition of NOX2 reduces locomotor impairment, inflammation, and oxidative stress after spinal cord injury. J Neuroinflammation. 2015;12:172.

38. Byrnes KR, Washington PM, Knoblach SM, Hoffman E, Faden Al. Delayed inflammatory mRNA and protein expression after spinal cord injury. J Neuroinflammation. 2011:8:130.

39. Bermudez S, Khayrullina G, Zhao Y, Byrnes KR. NADPH oxidase isoform expression is temporally regulated and may contribute to microglial/macrophage polarization after spinal cord injury. Mol Cell Neurosci. 2016;77:53-64.

40. Tian DS, Li CY, Qin C, Murugan M, Wu LJ, Liu JL. Deficiency in the voltagegated proton channel Hv1 increases M2 polarization of microglia and attenuates brain damage from photothrombotic ischemic stroke. J Neurochem. 2016;139:96-105.

41. Qin L, Liu Y, Wang T, Wei SJ, Block ML, Wilson B, Liu B, Hong JS. NADPH oxidase mediates lipopolysaccharide-induced neurotoxicity and proinflammatory gene expression in activated microglia. J Biol Chem. 2004;279:1415-21.

42. Novak ML, Koh TJ. Macrophage phenotypes during tissue repair. J Leukoc Biol. 2013;93:875-81.

43. Platanias LC. Mechanisms of type--- and type-II-interferon-mediated signalling. Nat Rev Immunol. 2005;5:375-86.

44. Padgett LE, Anderson B, Liu C, Ganini D, Mason RP, Piganelli JD, Mathews CE, Tse HM. Loss of NOX-derived superoxide exacerbates diabetogenic CD4 T-cell effector responses in type 1 Ddabetes. Diabetes. 2015:64:4171-83.

45. Thomas KE, Galligan CL, Newman RD, Fish EN, Vogel SN. Contribution of interferon-beta to the murine macrophage response to the toll-like receptor 4 agonist, lipopolysaccharide. J Biol Chem. 2006;281:31119-30.

46. Couper KN, Blount DG, Riley EM. IL-10: the master regulator of immunity to infection. J Immunol. 2008;180:5771-7.

47. Curtale G, Mirolo M, Renzi TA, Rossato M, Bazzoni F, Locati M. Negative regulation of toll-like receptor 4 signaling by IL-10-dependent microRNA146b. Proc Natl Acad Sci U S A. 2013;110:11499-504.

48. Berg DJ, Kuhn R, Rajewsky K, Muller W, Menon S, Davidson N, Grunig G, Rennick D. Interleukin-10 is a central regulator of the response to LPS in murine models of endotoxic shock and the Shwartzman reaction but not endotoxin tolerance. J Clin Invest. 1995;96:2339-47.

49. Kelly B, Tannahill GM, Murphy MP, O'Neill LA. Metformin inhibits the production of reactive oxygen species from NADH: ubiquinone oxidoreductase to limit induction of interleukin-1 beta (IL-1 beta) and boosts interleukin-10 (IL-10) in lipopolysaccharide (LPS)-activated macrophages. J Biol Chem. 2015:290:20348-59.

50. Murray PJ. The primary mechanism of the IL-10-regulated antiinflammatory response is to selectively inhibit transcription. Proc Natl Acad Sci U S A. 2005;102:8686-91. 
51. Qin H, Holdbrooks AT, Liu Y, Reynolds SL, Yanagisawa LL, Benveniste EN SOCS3 deficiency promotes M1 macrophage polarization and inflammation. J Immunol. 2012;189:3439-48.

52. Csuka E, Morganti-Kossmann MC, Lenzlinger PM, Joller $\mathrm{H}$, Trentz $\mathrm{O}$, Kossmann T. IL-10 levels in cerebrospinal fluid and serum of patients with severe traumatic brain injury: relationship to IL-6, TNF-alpha, TGF-beta1 and blood-brain barrier function. J Neuroimmunol. 1999;101:211-21.

53. Woodcock T, Morganti-Kossmann MC. The role of markers of inflammation in traumatic brain injury. Front Neurol. 2013;4:18.

54. Knoblach SM, Faden Al. Interleukin-10 improves outcome and alters proinflammatory cytokine expression after experimental traumatic brain injury. Exp Neurol. 1998;153:143-51.

55. Perez-de Puig I, Miro F, Salas-Perdomo A, Bonfill-Teixidor E, Ferrer-Ferrer M, Marquez-Kisinousky L, Planas AM. IL-10 deficiency exacerbates the brain inflammatory response to permanent ischemia without preventing resolution of the lesion. J Cereb Blood Flow Metab. 2013;33:1955-66.

56. Grilli M, Barbieri I, Basudev H, Brusa R, Casati C, Lozza G, Ongini E. Interleukin-10 modulates neuronal threshold of vulnerability to ischaemic damage. Eur J Neurosci. 2000;12:2265-72.

57. Qian L, Block ML, Wei SJ, Lin CF, Reece J, Pang H, Wilson B, Hong JS, Flood PM. Interleukin-10 protects lipopolysaccharide-induced neurotoxicity in primary midbrain cultures by inhibiting the function of NADPH oxidase. J Pharmacol Exp Ther. 2006;319:44-52.

58. Oliva Jr AA, Kang Y, Sanchez-Molano J, Furones C, Atkins CM. STAT3 signaling after traumatic brain injury. J Neurochem. 2012;120:710-20.

59. Zhao JB, Zhang Y, Li GZ, Su XF, Hang CH. Activation of JAK2/STAT pathway in cerebral cortex after experimental traumatic brain injury of rats. Neurosci Lett. 2011:498:147-52.

60. Yamauchi K, Osuka K, Takayasu M, Usuda N, Nakazawa A, Nakahara N, Yoshida M, Aoshima C, Hara M, Yoshida J. Activation of JAK/STAT signalling in neurons following spinal cord injury in mice. J Neurochem. 2006;96: 1060-70.

61. Satriotomo I, Bowen KK, Vemuganti R. JAK2 and STAT3 activation contributes to neuronal damage following transient focal cerebral ischemia. J Neurochem. 2006;98:1353-68.

62. Shechter R, London A, Varol C, Raposo C, Cusimano M, Yovel G, Rolls A, Mack M, Pluchino S, Martino G, et al. Infiltrating blood-derived macrophages are vital cells playing an anti-inflammatory role in recovery from spinal cord injury in mice. PLoS Med. 2009:6:e1000113.

63. Ma SF, Chen YJ, Zhang JX, Shen L, Wang R, Zhou JS, Hu JG, Lu HZ. Adoptive transfer of $\mathrm{M} 2$ macrophages promotes locomotor recovery in adult rats after spinal cord injury. Brain Behav Immun. 2015;45:157-170.

\section{Submit your next manuscript to BioMed Central and we will help you at every step:}

- We accept pre-submission inquiries

- Our selector tool helps you to find the most relevant journal

- We provide round the clock customer support

- Convenient online submission

- Thorough peer review

- Inclusion in PubMed and all major indexing services

- Maximum visibility for your research

Submit your manuscript at www.biomedcentral.com/submit 